\title{
НАБЛЮДАЕТСЯ ЛИ КОНВЕРГЕНЦИЯ РОЖДАЕМОСТИ В СТРАНАХ-ЧЛЕНАХ ЕВРОПЕЙСКОГО СОЮЗА? *
}

\author{
ДЖАМПАОЛО ЛАНЦИЕРИ
}

\begin{abstract}
В статье показывается важность учета дополнительных ограничений при разработке прогнозных гипотез для группы стран. В случае рождаемости, рассматриваемой в данной статье, это может быть выражено в виде конвергенции демографического поведения стран в будущем, что должно найти отражение в прогнозных гипотезах. Такое «международное» ограничение может иметь как теоретические, так и эмпирические основания.

В статье представлен анализ различных концепций конвергенциии, предложенных в литературе, а также соответствующие показатели. Предложен авторский метод анализа и новый простой индикатор конвергенции, используемый для оценки наличия конвергенции в 27 странах ЕС. Автор анализирует возможное влияние присоединения новых стран к ЕС на конвергенцию в рождаемости. В заключении обсуждается возможность использования предложенного подхода при разработке прогнозов.
\end{abstract}

Ключевые слова: конвергенция в рождаемости, Европейский Союз, расширение ЕС, прогнозные гипотезы в рождаемости.

\section{1 . ВВЕДЕНИЕ}

Демографические прогнозы - один из основных результатов демографических исследований. Как правило, они разрабатываются национальными статистическими службами, научно-исследовательскими институтами и международными организациями. Когда прогнозы составляются для набора территориальных единиц (регионы, страны, регионы мира и т.д. ${ }^{1}$ ), то дополнительным требованием становится согласованность этих прогнозов между собой. Фактически создание прогнозов для нескольких территориальных единиц предполагает добавление поперечного среза к обычному анализу временных рядов. Например, если в странах наблюдаются схожие демографические тенденции, эта дополнительная информация должна быть принята во внимание при разработке прогнозных гипотез.

\footnotetext{
ДЖАМПАОЛО ЛАНЦИЕРИ, ЕВРОПЕЙСКАЯ КОМИССИЯ - ЕВРОСТАТ, ОТДЕЛ НАРОДОНАСЕЛЕНИЯ. ЛЮКСЕМБУРГ. E-MAIL: giampaolo.lanzieri@ec.europa.eu.

МНЕНИЕ, ВЫРАЖЕННОЕ В ЭТОЙ СТАТЬЕ, ПРИНАДЛЕЖИТ ИСКЛЮЧИТЕЛЬНО АВТОРУ И НЕ ОБЯЗАТЕЛЬНО ОТРАЖАЕТ ТОЧКУ ЗРЕНИЯ ЕВРОПЕЙСКОЙ КОМИССИИ.

* ПЕРЕВОД ОРИГИНАЛЬНОЙ СТАТЬИ LANZIERI GIAMPAOLO (2010). IS FERTILITY CONVERGING ACROSS THE MEMBER STATES OF THE EUROPEAN UNION? // WORK SESSION ON DEMOGRAPHIC PROJECTIONS. LISBON, 28-30 APRIL 2010. LUXEMBOURG: PUBLICATIONS OFFICE OF THE EUROPEAN UNION. ПЕРЕВОД ВЫПОЛНЕН ЕКАТЕРИНОЙ ПЕТУХОВОЙ.
}

\footnotetext{
${ }^{1}$ Для простоты я рассматриваю здесь только страны.
} 
Типичными примерами возможных ограничений служат теории первого и второго демографических переходов. Первая теория объясняет снижение вначале смертности, а затем рождаемости до более низкого уровня; вторая фокусирует внимание на изменениях в семье и рождаемости в более широком социальном и культурном контексте. Таким образом, если движущей силой первого демографического перехода является смертность, то второго - рождаемость [Van de Kaa 2004]. Теория первого демографического перехода общепризнана в научной литературе, в то время как вклад теории второго демографического перехода в понимание демографических изменений все еще подвергается сомнению [Coleman 2004]. Идея конвергенции встроена в теорию демографического перехода.

В отличие от обширной экономической литературы, в которой конвергенция выводится из неоклассической модели роста Солоу, причем с ней связывается ряд политических последствий, конвергенции в демографии с точки зрения эмпирических доказательств до сих пор уделялось относительно мало внимания. Между тем, если демографическая конвергенция между странами имеет место, то это создает теоретические предпосылки, полезные для разработки прогнозных гипотез, а «международная» согласованность результатов обеспечивается благодаря их использованию. Поэтому в настоящей статье я обращаюсь к вопросу о конвергенции рождаемости в группе из 27 европейских стран, входящих в настоящее время в Европейский Союз (ЕС).

В широком социальном смысле существуют несколько индикаторов, описывающих конвергенцию в Европе. По мнению ряда исследователей, в течение прошлого столетия в западноевропейских обществах шел процесс социальной интеграции [Kaelble 1990]. Даже если в странах, недавно присоединившихся к ЕС, он пока не очевиден, сближение с европейской социальной моделью вполне возможно в будущем [Draxler, Van Vliet 2010]. Сосредоточивая внимание на демографическом аспекте, Уоткинс [Watkins 1990] показал, что в течение XIX и XX веков наблюдалась тенденция к большей демографической однородности регионов, находящихся в общих национальных границах.

Говоря о странах-членах ЕС, можно с уверенностью предположить, что общая политика и обмен успешным опытом могут способствовать конвергенции в ряде областей. Вообще, конвергенция представляет собой центральную концепцию в политике ЕС на многих направлениях. Например, основная цель Структурных фондов, одного из крупнейших каналов финансирования в ЕС, заключается в сокращении разрыва между уровнями развития различных регионов ЕС (цель, обозначенная как «конвергенция») благодаря усилению их социального единства и повышению экономического благосостояния. Конвергенция, следовательно, - это естественная концептуальная основа для формирования гипотез, относящихся к ЕС.

Первая гипотеза, которую мы рассмотрим, состоит в том, что демографическая конвергенция наблюдается в Европе в целом, независимо от того, является ли страна членом ЕС или нет. Мы ограничимся здесь рассмотрением рождаемости, так как рождение ребенка это в основном результат индивидуального решения, а рождаемость имеет основополагающее значение для теории второго демографического перехода, который, как 
предполагается, совершается в наши дни в Европе. Забегая вперед, замечу, что следующая гипотеза, требующая проверки, заключается в том, могут ли политические усилия, предпринимаемые на уровне ЕC и направленные на социально-экономическую конвергенцию стран-членов ЕС, дать дополнительный импульс или даже вызвать конвергенцию рождаемости.

Статья построена следующим образом: в разделе 2 я представляю различные концепции конвергенции, предложенные в литературе, а также соответствующие показатели; в разделе 3 излагается мой метод анализа и предлагается новый простой индикатор конвергенции; в разделе 4, используя обычные методы, я оцениваю наличие конвергенции в 27 странах, входящих в настоящее время в ЕС; в разделе 5 с помощью предложенного в разделе 3 индикатора я анализирую с точки зрения конвергенции рождаемости наблюдавшиеся в прошлом возможные последствия вступления в ЕС новых членов; в разделе 6 обсуждается возможность использования предложенного подхода при разработке прогнозов.

\section{2. ОПРЕДЕЛЕНИЯ И ИЗМЕРИТЕЛИ КОНВЕРГЕНЦИИ}

В экономической литературе можно найти несколько определений конвергенции [Sala-iMartin 1995]. Первая наиболее широко используемая концепция описывает конвергенцию набора географических единиц (стран, регионов, графств и т.д.) как сокращение во времени дисперсии того или иного показателя (например, ВВП на душу населения), измеряемой, как правило, с помощью стандартного отклонения или схожих мер ${ }^{2}$. Это называется $\sigma$ конвергенцией, она, таким образом, проявляется в сокращении со временем межстранового разброса показателя. Следовательно, $\sigma$-конвергенция наблюдается, если:

$$
\sigma_{\mathrm{t}}>\sigma_{\mathrm{t}+\mathrm{T}}
$$

где $\sigma_{t}$ представляет собой стандартное отклонение (или связанную с ней меру) показателя в момент времени $t$.

Другая распространенная концепция носит название $\beta$-конвергенции. Это понятие введено в работах Барро и Сала-и-Мартина [Barro, Sala-i-Martin 1992], которые рассматривали зависимость между средними темпами роста ВВП на душу населения в течение некоторого времени и логарифмом его исходного уровня:

$$
\gamma_{i, t, t+T}=\alpha+\beta \cdot \ln \left(y_{i, t}\right)+\varepsilon_{i, t}
$$

где $\gamma_{i, t, t+T}=\ln \left(y_{i, t+T} / y_{i, t}\right) / T$ - среднегодовой темп роста переменной $y$ в стране $i$ в рассматриваемый период времени $(t, t+T) ; y_{i, t}$ - значение показателя в начальный момент времени $t$, а $\varepsilon$ i,t - случайные ошибки. Модель (2) часто называют регрессией Барро, в ней

\footnotetext{
2 Обычно в литературе встречаются как показатель стандартного отклонения логарифма исходного показателя, так и коэффициент вариации самого показателя. Оба инвариантны по отношению к среднему значению. Иногда используется стандартное отклонение самого показателя.
} 
отрицательный угловой коэффициент означает, что показатель на территориях с более низким исходным значением $y$ вырос за рассматриваемый период сильнее, чем там, где исходное значения $y$ в начальный момент времени было выше. Таким образом, $\beta$ конвергенция предполагает сближение как результат различий в средней скорости роста: более высокой у догоняющих стран с низким изначальным уровнем и более низкой у стран, ушедших вперед и имеющих высокие исходные показатели. Янг с коллегами [Young et al. $2008]$ показали, что $\beta$-конвергенция является необходимым, но не достаточным условием для $\sigma$-конвергенции; напротив, $\sigma$-конвергенция является достаточным, но не необходимым условием $\beta$-конвергенции [Sala-i-Martin 1995].

Несмотря на критику, появлявшуюся, начиная с Фридмана [Friedman 1992] и Кью [Quah 1993], в экономической литературе этот подход и его последующие модификации получили широкое распространение. Для того чтобы устранить зависимость углового коэффициента в модели (2) от продолжительности периода анализа, Сала-и-Мартин [Salai-Martin 1995] предлагает также нелинейную модель ${ }^{3}$ для проверки на наличие $\beta$ конвергенции:

$$
\gamma_{i, t, t+T}=\alpha-\left(\frac{1-e^{-\lambda \cdot \mathrm{T}}}{T}\right) \cdot \ln \left(y_{i, t}\right)+\varepsilon_{i, t}
$$

где $\lambda$ можно интерпретировать как скорость конвергенции. Сала-и-Мартин [Sala-i-Martin 1994] проанализировал эти скорости для экономик нескольких территорий и утверждает, что они составляют приблизительно $2 \%$ в год, таким образом подтверждая идею чего-то вроде «естественного темпа конвергенции». Это утверждение было проверено Абреу с соавторами [Abreu et al. 2005] на основе метоанализа большого объема литературы, и они пришли к выводу, что скорость конвергенции довольно чувствительна к спецификации модели. Если принять во внимание неучитываемую неоднородность (например, в технологических уровнях), темп конвергенции, как правило, оказывается более высоким.

Когда анализ выполняется на уровне стран, может быть затруднительным принять гипотезу, что все страны обладают одинаковыми технологиями и преимуществами; тем не менее каждая страна может все же приближаться к иному устойчивому состоянию, но общим для всех темпом. Эта концепция носит название условной $\beta$-конвергенции, она может быть обнаружена с включением в спецификацию регрессии Барро дополнительного набора объясняющих переменных, предназначенных для учета различных технологий и преимуществ. Если конвергенция происходит между подгруппами стран, это называют клубной конвергенцией. Конвергенционные клубы, как правило, определяют с помощью фиктивных переменных, включенных в модель условной конвергенции. Кроме того, Аццони с коллегами [Azzoni et al. 2003] обращают внимание на важность исследования

\footnotetext{
3 Уравнение можно решить с использованием нелинейных методов. Также возможно решить его, используя обычный метод наименьших квадратов и затем получая $\beta$-параметр с использованием для преобразования формулы $\lambda=-1 / T[\ln (1+\beta \cdot T)]$. Однако, когда период времени велик и/или отрицательная величина оцениваемого наклона в абсолютном выражении высока, этот подход может привести к появлению отрицательных значений логарифма и поэтому не может быть использован.
} 
конвергенции с использованием микроданных вместо макроданных, поскольку на последние могут оказать влияние структурные смещения. Коул и Ноймайер [Cole, Neumayer 2003] подчеркивают необходимость взвешивания по численности населения в исследованиях по распределению доходов.

Учитывая критику способности модели (2) оценивать $\beta$-конвергенцию, Бойл и Маккарти [Boyle, McCarthy 1997] предлагают простую меру, направленную на фиксацию степени подвижности со временем внутри распределения (intra-distributional mobility). Для этого используется индикатор на основе коэффициента ранговой конкордации Кендалла $(\mathrm{RC})$ :

$$
R C_{t}=\frac{\operatorname{Var} \sum_{t=o}^{T} R_{i t}}{\operatorname{Var}\left[(T+1) \cdot R_{i 0}\right]}
$$

где $R_{i t}$ - коэффициент для страны $i$ в момент времени $t$. Эти авторы также предлагают использовать двухгодичную версию многолетнего коэффициента RC в (4):

$$
R C a_{t}=\frac{\operatorname{Var}\left(R_{i 0}+R_{i t}\right)}{\operatorname{Var}\left(2 \cdot R_{i 0}\right)}
$$

Значения этих коэффициентов могут находиться в диапазоне от нуля до единицы: чем ниже значение, тем больше степень мобильности в рамках распределения.

В литературе предложены и другие концепции конвергенции и соответствующие методы ее измерения. Например, Маэсо-Фернандес [Maeso-Fernandez 2003] применяет анализ временных рядов для изучения разрыва между рядом стран и США. Лаурини с коллегами [Laurini et al. 2005] используют непараметрические методы для определения конвергенционных клубов по доходам в муниципалитетах Бразилии. Томлянович и Фогельзанг [Tomljanovich, Vogelsang 2002] применяют иной эконометрический подход для оценки конвергенции регионов США по ВВП. Филлипс и Сул [Phillips, Sul 2007] разрабатывают новый метод, который позволяет оценивать конвергенцию и клубную конвергенцию с помощью регрессионной модели.

Регрессия Барро, опирающаяся на среднегодовой темп роста по отношению к исходному уровню, на самом деле маскирует любые вариации внутри рассматриваемого периода. Поэтому в некоторых исследованиях период разбивается на подпериоды для оценки рассматриваемой переменной на отдельных интервалах. Это относится и к анализу на основе эконометрического подхода, где наличие структурных интервалов может повлиять на выполнение теста на единичные корни.

\section{1. Некоторые исследования конвергенции рождаемости}

По сравнению с исследованиями конвергенции в области экономики число исследований конвергенции в рождаемости относительно невелико. Уилсон [Wilson 2001], используя показатели, основанные на распределении мирового населения по суммарной рождаемости в определенные моменты времени, выявил наличие конвергенции на мировом уровне во 
второй половине прошлого века. Он отметил, что экономические различия между бедными и богатыми странами приобретают все меньшее значение для демографии и что демографическая конвергенция может рассматриваться как один из элементов социальнодемографических изменений, которые, вероятно, опережают экономическое развитие. В то же время Дориус [Dorius 2008] утверждает, что имеется больше доказательств в пользу дивергенции, нежели конвергенции. Для измерения межстрановых различий в рождаемости он сосредоточил внимание на относительных, а не абсолютных различиях в показателях интенсивности деторождения. В частности, он использовал три индекса неравенства (коэффициент Джини, среднее логарифмическое отклонение и индекс Тейла), чтобы выявить источник изменений в распределении стран по уровню рождаемости. Результаты проведенного анализа, основанного на расчетах взвешенных по численности населения показателей $\sigma$ - и $\beta$-конвергенции и неравенства, показывают, что конвергенция началась только в конце периода 1955-2005 гг., из чего Дориус сделал вывод, что вторую половину XX века нельзя считать периодом конвергенции рождаемости в глобальном масштабе. Для того чтобы разделить экономический и демографический эффекты, Хербертссон с коллегами [Herbertsson et al. 2000] сосредоточили внимание на условной модели и нашли доказательства как абсолютной, так и условной конвергенции для коэффициентов рождаемости, рассчитанных примерно для 190 стран в период между 1978 и 1998 г.

Томка [Tomka 2002], используя другой подход, анализировал демографическую конвергенцию между конкретной страной (Венгрией) и группой стран (Западная Европа). Для этого он предложил индексы, основанные на стандартизованных отличиях от средних показателей западноевропейских стран, и пришел к выводу, что Венгрия сближалась с этими странами с начала до середины прошлого века, после чего, начиная с середины 1960 х годов, последовал период дивергенции.

Другие исследования, напротив, относятся к региональному анализу в пределах одной страны. Например, Франклин [Franklin 2002; 2003], рассматривая $\sigma$-конвергенцию, обнаружил, что фактически региональная рождаемость в Италии расходилась с момента объединения до начала послевоенного периода, затем последовал период конвергенции до 1970 -х годов, а потом снова начался период дивергенции. В то же время, анализируя $\beta$ конвергенцию, тот же автор пришел к выводу, что после Второй мировой войны отмечалась конвергенция рождаемости в регионах Италии со скоростью более $2 \%$ и что учет пространственной зависимости существенно не улучшил модель на региональном уровне (в то время как это произошло при дальнейшем анализе на уровне провинций). Для другой средиземноморской страны (Греции) Котзаманис и Дюкенн [Kotzamanis, Duquenne 2006] нашли доказательства конвергенции демографических структур в ее регионах; в частности, это касается периодных и когортных показателей рождаемости, демонстрирующих ясную тенденцию к сближению.

Еще одна область исследований в демографии касается конвергенции между группами населения в пределах определенной территориальной единицы. К ним относится, например, анализ тенденции различий в демографическом поведении между этническими 
группами или между местными жителями и иностранцами [Haines 2002]. В этой статье данный вопрос не рассматривается.

\section{3. ДАННЫЕ И МЕТОД АНАЛИЗА}

Источником используемых в анализе данных в основном являются национальные статистические службы, передающие их в Евростат (информация размещена в свободном доступе в базе данных Евростата). Официальные статистические данные дополнены авторскими оценками. К 27 государствам-членам ЕС относятся следующие страны ${ }^{4}$ : Бельгия (BE), Болгария (BG), Чехия (CZ), Дания (DK), Германия (DE), Эстония (EE), Ирландия (IE), Греция (EL), Испания (ES), Франция (FR), Италия (IT), Кипр (CY), Латвия (LV), Литва (LT), Люксембург (LU), Венгрия (HU), Мальта (MT), Нидерланды (NL), Австрия (АT), Польша (PL), Португалия (PT), Румыния (RO), Словения (SI), Словакия (SK), Финляндия (FI), Швеция (SE) и Соединенное Королевство (UK). Данные по Франции относятся к метрополии и не включают заморские владения Франции. Данные по Кипру относятся к территории, контролируемой Правительством с 1974 г., а данные для Германии всегда включают Восточную Германию.

Как показано выше, конвергенция - многомерное понятие. Для проверки первой гипотезы, указанной в разделе 1, необходимо установить наличие конвергенции рождаемости независимо от формы, в которой она проявляется, например, в терминах сжатия межстранового распределения или подвижек внутри распределения. Поэтому я анализирую наличие $\sigma$-конвергенции, абсолютной $\beta$-конвергенции и $\gamma$-конвергенции используя простейшие методы оценки, то есть с помощью показателей, представленных в формулах (1), (2), (4) и (5). Поскольку рождаемость в отличие от смертности не является фатальным событием и может повторяться, я использую два показателя: коэффициент суммарной рождаемости (КСР) и средний возраст материнства (CBM). Первый из них - это сумма возрастных коэффициентов рождаемости в данном году, а второй средневзвешенний возраст в рамках того же распределения. Иными словами, КСР показывает интенсивность рождаемости, в то время как СВМ отражает её тайминг [Vallin, Caselli 2006]. Таким образом, цель анализа конвергенции расширяется и охватывает не только уровень, но и тайминг рождаемости.

Обнаружение абсолютной $\beta$-конвергенции предполагает, что все страны достигнут одного и того же долгосрочного равновесия (устойчивого состояния, в экономических терминах) в одно и тоже время. Использование КСР для анализа $\beta$-конвергенции имеет интересную интерпретацию с демографической точки зрения. Как известно [Preston et al.

\footnotetext{
${ }^{4}$ Страны перечислены согласно официальному протокольному порядку ЕС в порядке английского алфавита и с их официально принятыми сокращениями.

5 Для того чтобы отличить коэффициент регрессии Барро от коэффициентов ранговой конкордации, я следую предложению Бойла и Маккарти [Boyle, McCarthy 1999] для обозначения последних как $\gamma$-конвергенция.
} 
2001], в стабильном населении истинный коэффициент естественного прироста $r$ может быть выражен с помощью формулы:

$$
r=\frac{\ln \kappa \mathrm{CP}+\ln S+p\left(A_{M}\right)}{G},
$$

где $S$ - доля девочек среди родившихся, одинаковая у матерей всех возрастов, $p\left(A_{M}\right)-$ вероятность дожить от рождения до среднего возраста матери и $G$ - средняя длина поколения. При условии постоянства значения этих компонент во временном диапазоне $\left(t_{1}, t_{2}\right)$ изменение истинного коэффициент естественного прироста в период $\left(t_{1}, t_{2}\right)$ можно представить в виде:

$$
\Delta r=\frac{\ln \left(\frac{\mathrm{KCP}_{2}}{\mathrm{KCP}_{1}}\right)}{G} .
$$

Если принять эти упрощения, то зависимая переменная в регрессии Барро, измеряющая $\beta$ конвергенцию КСР, на самом деле пропорциональна ${ }^{6}$ изменению истинного коэффициента естественного прироста соответствующего стабильного населения.

Для оценки второй гипотезы, рассматривающей влияние вступления в ЕС на демографическое поведение новых государств-членов, индексы, используемые здесь для общего анализа конвергенции, возможно, не соответствуют «целевому назначению». В этом случае интерес представляет не конвергенция между странами, а движение группы стран (вновь присоединившихся) в направлении государств, уже бывших членами ЕС. В рамках этого теоретического подхода предполагается, что «старые» государства-члены ЕС разделяют общие ценности, к которым должны приблизиться и «новые» члены Союза. Если не говорить о некоторых эконометрических методах, не рассматриваемых в этой работе, для наших целей можно использовать показатель, предложенный Томкой [Tomka 2002]:

$$
z=\frac{x-\mu}{\sigma}
$$

где $x$ - значение для рассматриваемой страны, а $\mu$ и $\sigma$-соответственно среднее значение и стандартное отклонение для группы стран, конвергенция с которыми должна быть оценена. Естественной модификацией этого индекса в случае, когда анализируется более одной страны, может быть расчет среднего значения из набора страновых индексов:

$$
Z=\sum_{i=1}^{k} Z_{i}=\sum_{i=1}^{k} \frac{x_{i}-\mu}{\sigma}
$$

Однако такой показатель чувствителен к изменениям в дисперсии внутри референтной группы. В центре же моего внимания стоит процесс конвергенции «новых» государствчленов независимо от того, наблюдается ли конвергенция или дивергенция среди «старых» государств-членов ЕС. Я рассматриваю эту форму конвергенции к «внешнему» для набора

\footnotetext{
${ }^{6} \mathrm{C}$ коэффициентом пропорциональности G/T, который может быть равен единице, если период анализа
} выбран равным средней длине поколения. 
рассматриваемых стран значению, наблюдаемому или теоретическому, как относительную конвергенциию.

Первым простым решением этой проблемы может быть обычный коэффициент вариации, но вычисленный по значениям для каждой из вновь присоединенных стран и агрегированному значению для ЕС, состоящего из «старых» членов (до вступления «новых» государств в ЕC). Я обозначаю этот показатель как $\mathrm{CV}^{*}$, чтобы он отличался от коэффициента вариации $\mathrm{CV}$, рассчитанного для полного набора стран. С этой точки зрения изменчивость внутри ЕС до включения новых членов можно рассматривать как вариабильность регионов одной страны, чем, как правило, пренебрегают при оценке конвергенции между странами. Однако, устраняя изменчивость внутри группы «старых» государств-членов, эта мера не дает представления о конвергенции в направлении к ним.

Для построения простой меры относительной конвергенции я рассчитываю квадратичное отклонение каждой новой страны от значения для ЕС, полученного путем агрегирования исходных данных ${ }^{7}$ для «старых» членов ЕС, а затем усредняю его по числу случаев. Это не что иное, как дисперсия вокруг заданного значения, поэтому этот показатель может рассматриваться так же, как и показатели, используемые в анализе $\sigma$ конвергенции. Для каждой временной точки $t$ анализируемого периода я определяю таким образом индекс относительной конвергенции (ИОК):

$$
\text { ИОК }_{t}=\sqrt{\text { ИОК }_{t}^{2}}=\sqrt{\sum_{i=1}^{n} \frac{\left(x_{i, t}-\mu_{t}\right)^{2}}{n}}
$$

и коэффициент относительной конвергенции (КОК):

$$
\mathrm{KOK}_{t}=\frac{\text { иОК }_{t}}{\mu_{t}}
$$

где $\mu_{t}$ - среднее значение для ЕС, рассматриваемого в составе только «старых» государствчленов. Если посмотреть на временные ряды этих оценок, то уменьшение значений указывает на конвергенцию относительно рассматриваемого значения и наоборот. Если исследуется конвергенция относительно остальных стран ЕС только одной страны, то ИОК сводится к простой разнице между значениями для этой страны и другими странами ЕС, а КОК - к относительной разнице.

ИОК имеет полезное свойство. Легко показать, что квадрат ИОК можно дезагрегировать следующим образом:

$$
\text { ИОК }_{t}^{2}=\sum_{i=1}^{n} \frac{\left(x_{i, t}-\mu_{t}\right)^{2}}{n}=\sum_{i=1}^{n} \frac{\left(x_{i, t}-\overline{x_{t}}\right)^{2}}{n}+\left(\overline{x_{t}}-\mu_{t}\right)^{2}=\sigma_{N}^{2}+\Delta^{2},
$$

где $x$ - это среднее по новым странам $N, \sigma_{N}^{2}$ - дисперсия и $\Delta$ - разница между средним

\footnotetext{
7 Если первоначальные данные недоступны, значения для ЕС могут быть получены путем усреднения значений для отдельных стран, взвешенных по численности их населения.
} 
значением для «новых» и агрегированным значением для «старых» государств-членов ЕС.

Эта декомпозиция позволяет понять, происходит ли конвергенция/дивергенция благодаря конвергенции внутри группы «новых» стран и/или благодаря сближению их средних значений со значением ЕС.

Очевидно, что ИОК и КОК могут быть использованы также при анализе конвергенции по отношению к заранее определенным значениям (например, теоретически определенным) или в исследованиях конвергенции между подгруппами населения (например, конвергенции в демографическом поведении местных уроженцев страны и приехавших в нее уроженцев других стран).

\section{4. КОНВЕРГЕНЦИЯ В 27 ЕВРОПЕЙСКИХ СТРАНАХ}

Из-за недостаточности данных их анализ для всего набора государств-членов ЕС-27 ограничивается периодом 1977-2007 гг. За эти 30 лет дисперсия КСР сократилась на четверть к началу 1990-х годов, после чего стабилизировалась примерно на уровне 0,155 . Что касается СВМ, то на протяжении того же периода вначале наблюдалась дивергенция, а затем с 1993 г. - конвергенция к начальному уровню дисперсии (рисунок 1).

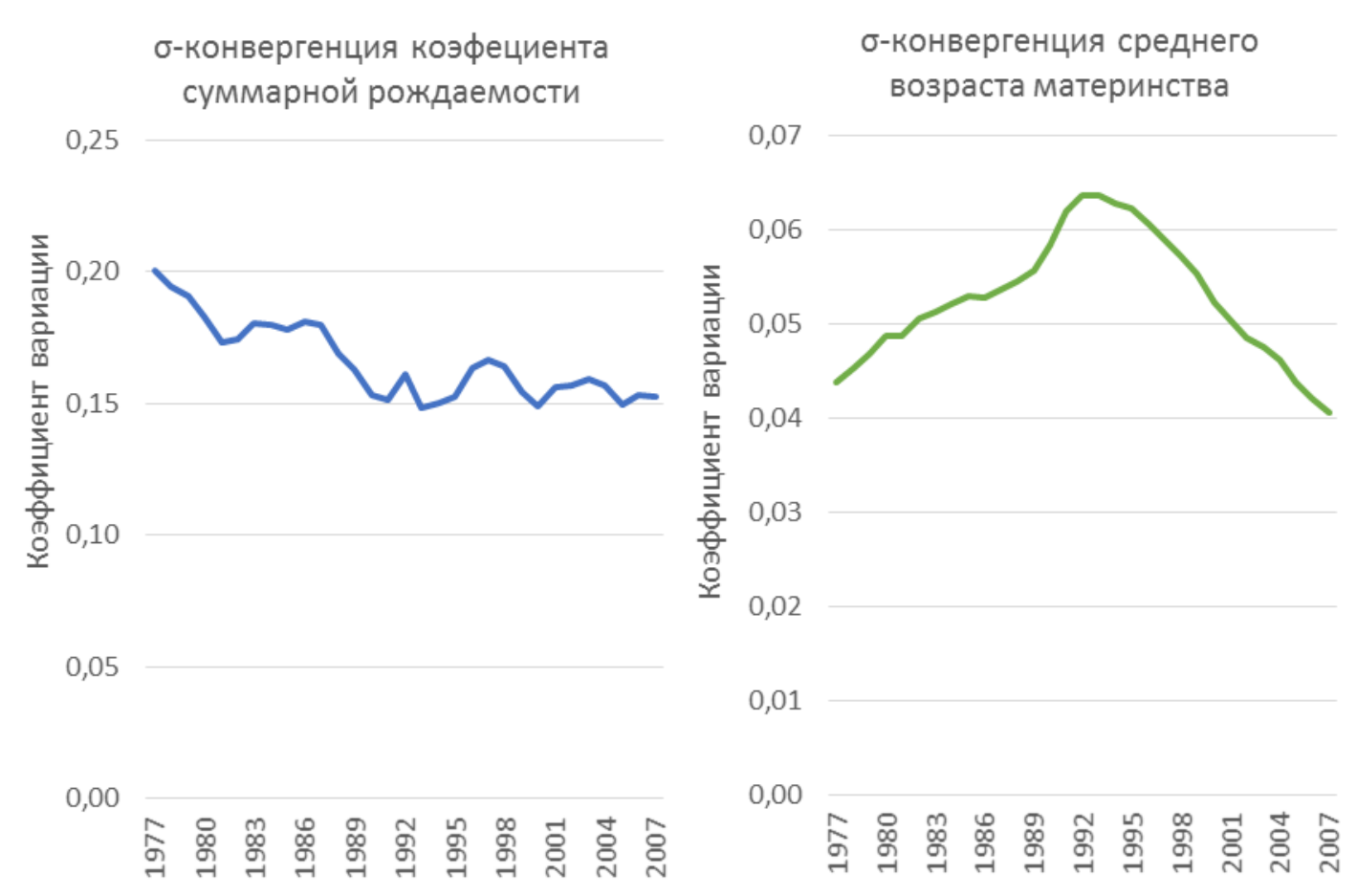

Рисунок 1. б-конвергенция показателей рождаемости в странах ЕС-27, 1977-2007 гг.

Таким образом, если судить по $\sigma$-конвергенции, то можно сказать, что до начала 1990-х годов сближались показатели интенсивности, а после этого - показатели тайминга деторождения. 
Как видно из регрессии Барро (рисунок 2), угловой коэффициент модели абсолютной $\beta$-конвергенции отрицателен как для КСР, так и СВМ $(-0,042$ и -0,012 соответственно). Это означает, что в странах с более высоким начальным уровнем суммарной рождаемости его снижение идет быстрее (они догоняют страны с более низким его уровнем «сверху»), тогда как сближение показателей среднего возраста материнства не столь выражено и идет в противоположном направлении (страны с более низкими значениями СВМ оказываются «догоняющими снизу»).



Рисунок 2. ß-конвергенция показателей рождаемости в странах ЕC-27, 1977-2007 гг.

Впрочем, следует отметить, что за период 1977-2007 гг. оба показателя меняли направление: в среднем КСР главным образом уменьшался, но недавно снова начал расти, в то время как СВМ менялся в противоположном направлении - немного снизился в начале периода, а затем устойчиво увеличивался. Поэтому использование линейной модели в течение всего периода может быть неоправданным. Оценка скорости конвергенции СВМ в $1,5 \%$ в год означает, что время, необходимое для сокращения расстояния наполовину, составляет 45 лет. Для КСР подобные вычисления с помощью формулы преобразования невозможны. Для краткости в этой статье я не пытаюсь рассматривать подпериоды или более глубоко анализировать скорость конвергенции. Можно отметить, что $\beta$-конвергенция происходила независимо от наличия $\sigma$-конвергенции (первая - необходимое, но не достаточное условие для последней). Точно так же можно сказать, что всякий раз, когда имелась $\sigma$-конвергенция, была и $\beta$-конвергенция, поскольку первая - достаточное, но не необходимое условие для второй. В целом применение концепции и модели $\beta$ конвергенции к анализу конвергенции в рождаемости по-прежнему остается затруднительным и в дальнейшем не используется в данной статье. 
Анализ $\gamma$-конвергенции подтверждает, что в период между 1977 и 2007 г. наблюдались подвижки внутри распределения как для КСР, так и для СВМ. Чтобы подчеркнуть связь с $\sigma$-конвергенцией, значения двух коэффициентов конкордации RC (двухлетних и многолетних) и коэффициент вариации, нормированный по исходному значению, показаны на одном графике на рисунке 3. Для облегчения сравнения показателей рождаемости левая и правая панели на рисунке 3 имеют одинаковую шкалу. СВМ показывает меньшую подвижность в своем распределении, чем КСР. Таким образом, страновые показатели среднего возраста материнства «движутся» в большей степени вместе, а не перекрещиваются, как это происходит с КСР.

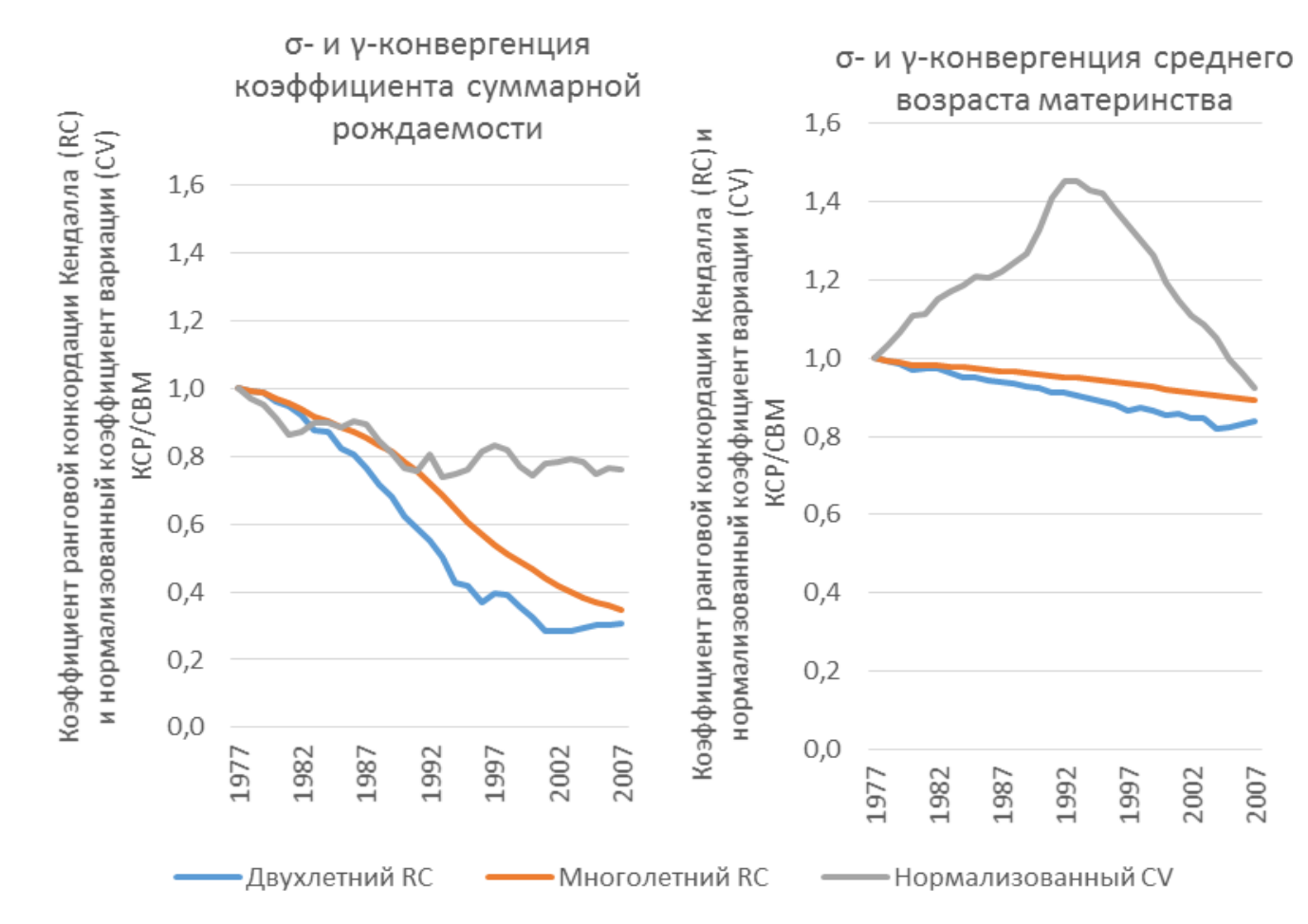

Рисунок 3. б-и $\gamma$-конвергенция показателей рождаемости в странах ЕС-27, 1977-2007 гг.

\section{5. ОТНОСИТЕЛЬНАЯ КОНВЕРГЕНЦИЯ И РАСШИРЕНИЕ ЕС}

Как показано выше, проверяемая здесь гипотеза состоит в том, что членство страны в ЕС способствует распространению действия демографических факторов, характерных для «старых» государств-членов ЕС, на вновь присоединенные страны, что подразумевает приближение их показателей рождаемости к соответствующим показателям стран ЕС. Чтобы проверить, подтверждалась ли эта гипотеза в прошлом, внимание сосредоточено на различных этапах укрупнения ЕС с целью найти эмпирические доказательства, подтверждающие гипотезу конвергенции между странами ЕС, особенно после появления его новых членов. Ниже рассматриваются ЕС-6, состоящий из 6 государств-членов, ЕС-9 в составе 9 государств-членов и так далее. В общей сложности рассматриваются 3 из 6 расширений ЕС, так как два последних произошли относительно недавно, что не позволяет 
судить о влиянии демографических трендов на «новые» страны, а два расширения ЕС были объединены для простоты (Греция в 1981 г. вместе с Испанией и Португалией в 1986 г.).

Полезно начать с анализа конвергенции рождаемости в 6 странах-основателях ЕС (Бельгия, Германия, Франция, Италия, Люксембург и Нидерланды). На рисунках 4 и 5 показаны соответственно тренды самих показателей рождаемости и коэффициентов их вариации. Тренды КСР и СВМ движутся скорее согласованно, чем вразнобой, или, иными словами, приближаются к одному и тому же уровню.

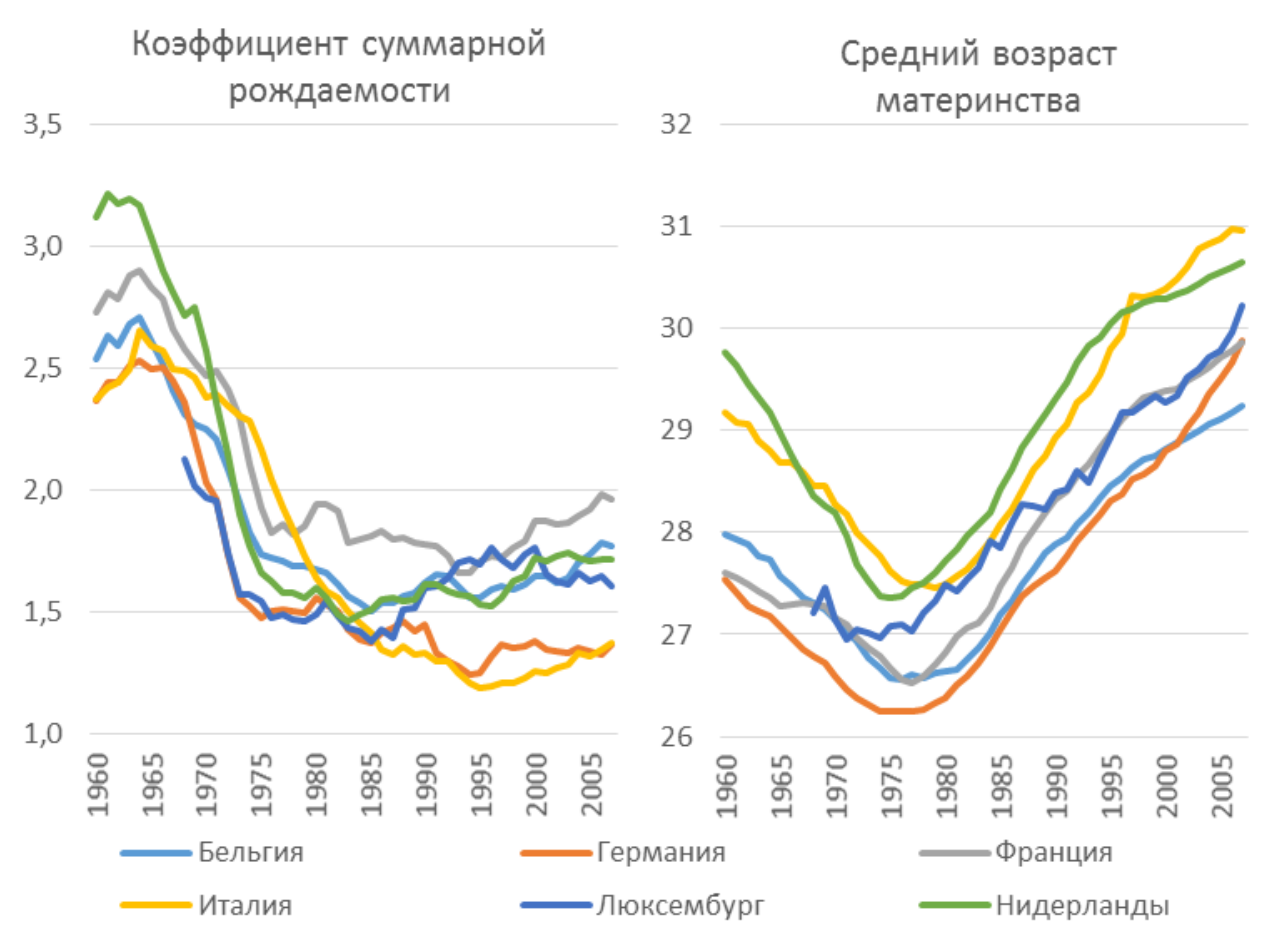

Рисунок 4. Показатели рождаемости в странах ЕC-6, 1960-2007




Рисунок 5. б-конвергенция рождаемости в странах ЕС-6, 1968-2007 гг.

Соответственно, рисунок 5 свидетельствует скорее о колебаниях, а не о постоянной тенденции к конвергенции/дивергенции во времени. Значение CV для КСР снижается до 0,08 в 1983 г. с пикового значения в 0,16, достигнутого десятью годами ранее (левая панель рисунка 5). Вслед за этим идет медленное восстановление до значений около 0,14. Если судить о конвергенции/дивергенции на основе снижения/увеличения коэффициента вариации, то строго говоря, убедительных доказательств наличия того или иного процесса на данном временном интервале для этих шести стран нет. Полученные результаты позволяют интерпретировать весь временной интервал и как период конвергенции с последующей небольшой дивергенцией, и как период долгосрочных колебаний вокруг среднего значения CV, равного 0,11. То же самое относится и к CBM: до 1975 г. наблюдалась конвергенция, сменившаяся длившейся 10 лет стабильностью с последующей дивергенцией до 1997 г., затем снова конвергенция. Но это, опять же, может быть истолковано и как колебания вокруг среднего значения $\mathrm{CV}$, равного 0,02.

В 1973 г. к ЕС присоединились Дания, Ирландия и Великобритания. Это первый случай относительной конвергенции, которая может быть проверена. К сожалению, данные за период до 1973 г. недоступны, что делает невозможным сравнение показателей до и после вступления этих стран в ЕС. Для облегчения интерпретации оценок $\sigma$-конвергенции и относительной конвергенции тренды КСР и СВМ также отображены на рисунке 6. Можно заметить, как показатели интенсивности и тайминга рождаемости в Ирландии приближаются к показателям в других странах ЕС. Эти тренды будут иметь очевидные последствия для оценки конвергенции.

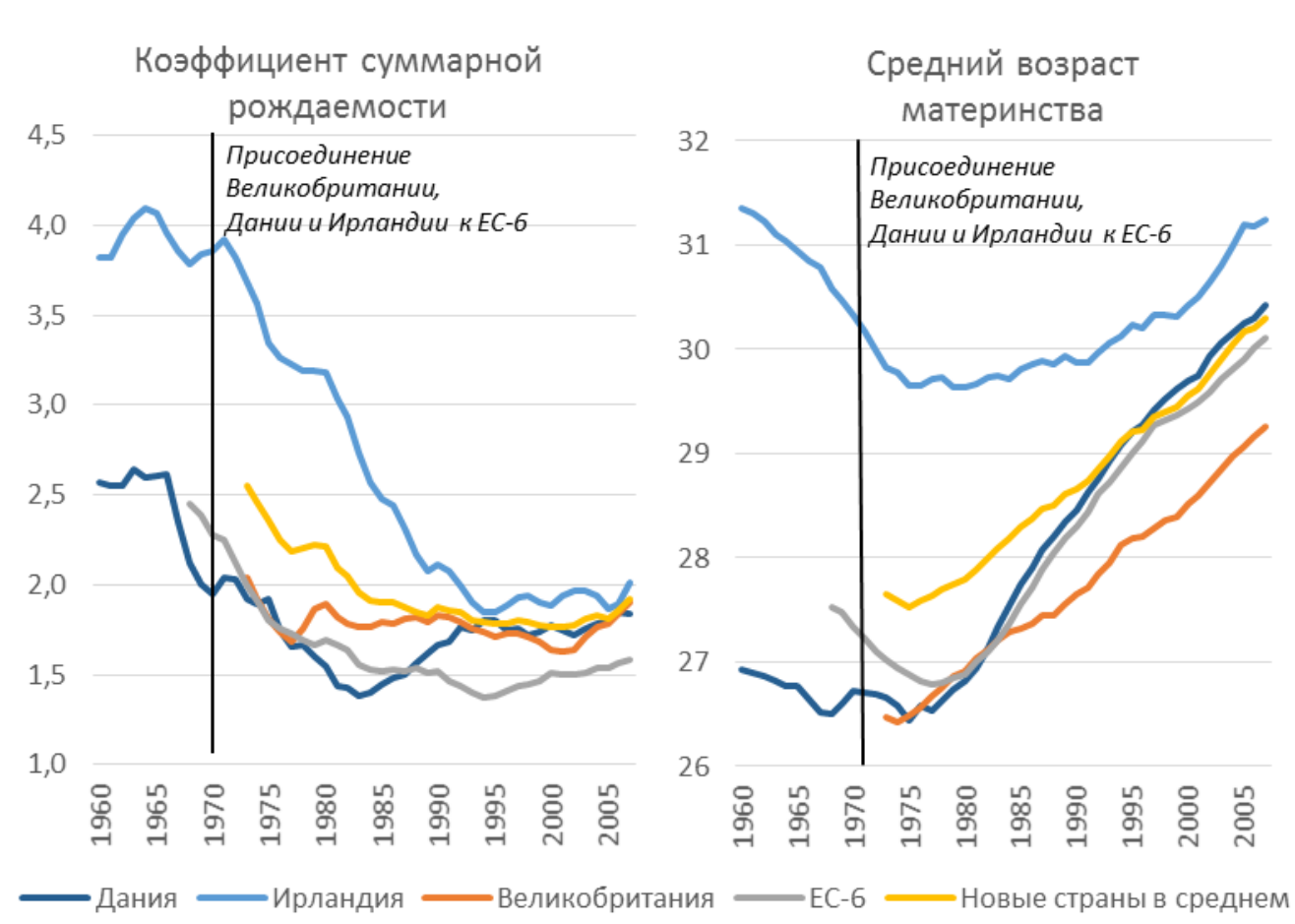

Рисунок 6. Показатели рождаемости в странах EC-9, 1960-2007 гг. 
Были рассчитаны две меры $\sigma$-конвергенции (рисунок 7). Первая - обычный коэффициент вариации CV для 9 государств-членов (6 членов-основателей и 3 страны, присоединившиеся в результате первого расширения); вторая мера (CV*) рассматривает государства-члены ЕС-6 как единое целое. Таким образом конвергенция рассчитывается для четырех территориальных единиц: для трех «новых» членов и «общий» для ЕС-6 в целом. Эти последние расчеты должны помочь оценить конвергенцию «новых» стран относительно ЕС в целом, устраняя эффект вариабельности среди «старых» государствчленов ЕС. Коэффициент вариации КСР (CV), рассчитанный для 9 стран ЕС, свидетельствует о четкой $\sigma$-конвергенцию вплоть до 1990 г., а затем о стабильности. Однако, если изменчивость в рамках ЕC-6 исключается с помощью расчета показателя CV*, то конвергенция КСР продолжается после 1990 г., пусть и с более умеренной скоростью. СВМ также сходится до 1994 г., а затем остается неизменным с обоими коэффициентами вариации чуть выше 0,02, которые на самом деле в несколько раз ниже, чем соответствующие значения для КСР.



Рисунок 7. б-конвергенция рождаемости в странах ЕС-9, 1973-2007 гг.

До сих пор конвергенция в ЕС-9 анализировалась без рассмотрения траектории движения «новых» стран по отношению к «старым». На рисунке 8 показаны коэффициенты относительной конвергенции, которые демонстрируют сильное снижение для КСР со значения 0,40 в 1979 г. до 0,15 в 2005 г. и сокращение вдвое значения для СВМ в течение десяти лет в 1980-е годы. На основании этого можно сделать вывод, что показатели рождаемости трех «новых» стран менялись в направлении сближения с другими государствами-членами ЕС. 


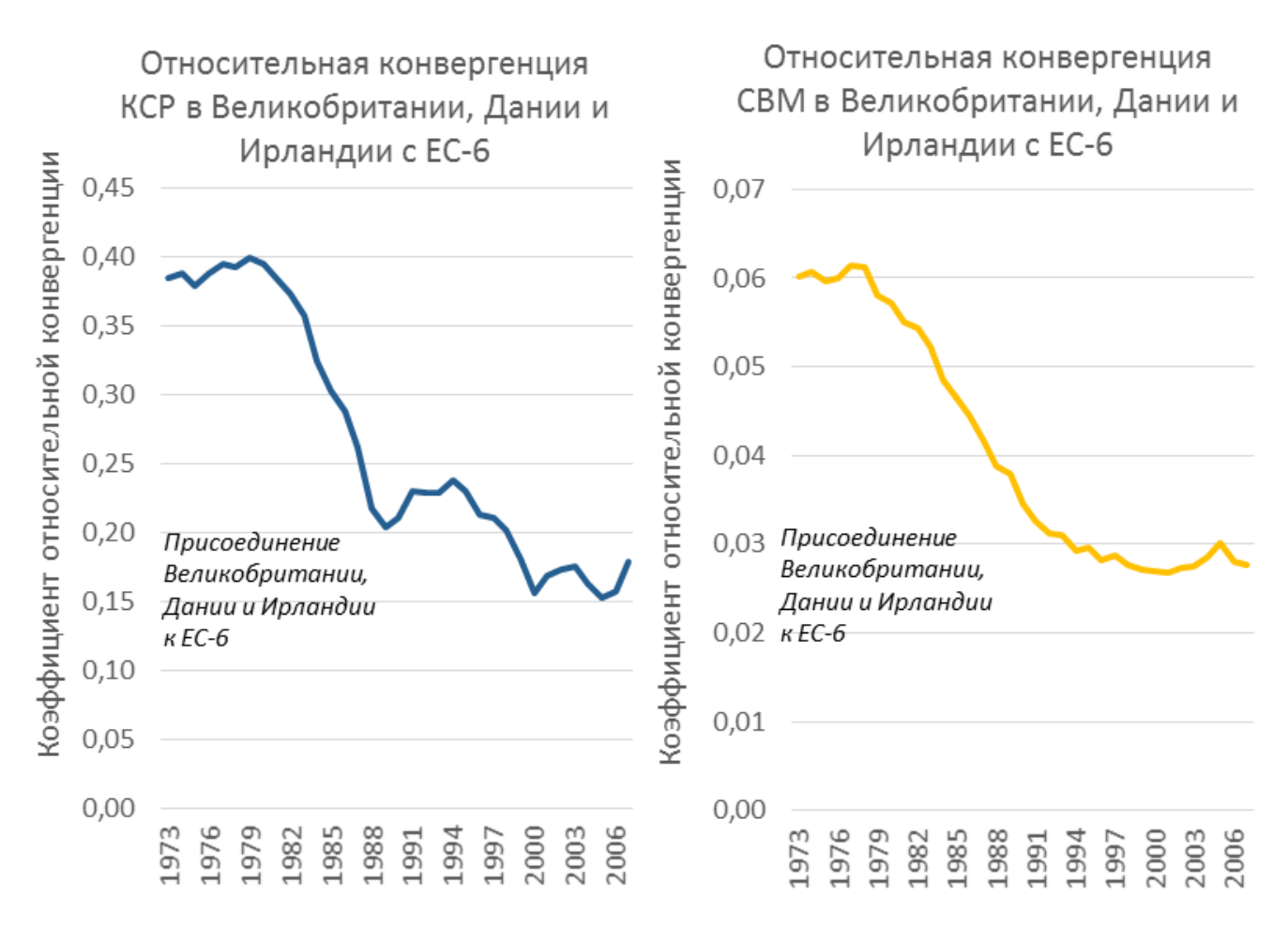

Рисунок 8. Относительная конвергенция показателей рождаемости Великобритании, Дании и Ирландии с показателями стран ЕС- 6, 1973-2007 гг.



Рисунок 9. Декомпозиция относительной конвергенции показателей рождаемости Великобритании, Дании и Ирландии с показателями стран ЕС-6, 1973-2007 гг. 
Рисунок 9 помогает понять причину сокращения коэффициента относительной конвергенции КОК. В случае с КСР сокращение до начала 1990-х годов происходило в основном за счет исчезновения расхождений между «новичками», а затем - за счет уменьшения различий между средними значениями для двух групп стран (старых и новых). Для СВМ - это одновременное сокращение различий и между «новичками», и между средними значениями вплоть до середины 1990-х годов, после чего стабильность КОК обусловлена устойчивостью первого из этих компонентов.

Для краткости я не останавливаюсь здесь подробно на других случаях расширения EC: в 1980-е годы за счет Греции, Испании и Португалии (рисунки 10-13), в 1995 г. за счет Австрии, Финляндии и Швеции (рисунки 14-17) и в середине 2000-х годов за счет оставшихся стран (рисунки 18-21). Отмечу лишь важнейшие элементы, касающиеся изменения демографического поведения стран после вступления в ЕС. После расширения ЕС с 9 до 12 стран отмечается относительная дивергенция КСР и с недавних пор относительная конвергенция для МАС. После расширения до 15 стран с недавних пор наблюдается относительная дивергенция КСР и умеренная относительная конвергенция МАС. Что касается недавнего расширения ЕС, то здесь нельзя еще сделать никаких выводов, так как прошло слишком мало времени, но анализируя последние 30 лет, можно отметить чередование относительной конвергенции и дивергенции для обоих показателей.

Таким образом, окончательных полных доказательств гипотезы, что членство в ЕС придает (дополнительный) толчок для конвергенции рождаемости относительно уровня $\mathrm{EC}$, не существует. Для интенсивности рождаемости, измеряемой КСР, характерна меньшая конвергенция в относительном выражении (на самом деле, в основном дивергенция), чем для темпа рождаемости. Даже когда кажется, что конвергенция налицо, обращение к предшествующему периоду показывает, что это вполне может быть просто продолжением тенденций, появившихся еще до вступления в ЕС. Тем не менее, хотя результаты, основанные на показателях, относящихся к прошлому, не полностью подтверждают гипотезу о конвергенции к стандартам ЕС, есть некоторые аргументы в пользу принятия этой гипотезы.

Прежде всего, в наше время все больше осознается значение влияния демографических тенденций и поэтому на них обращено большее внимание со стороны политиков. В частности, главами государств и правительств ЕС в 2007 г. было принято решение о создании Европейского Альянса в поддержку семей (European Alliance for Families), служащего платформой для обмена мнениями и опытом семейной политики и передовых практик между государствами-членами. Распространение успешного опыта политики, пытающейся оказывать влияние на демографические процессы государствчленов, может, таким образом, сделать ее более эффективной, чем в прошлом.

Кроме того, нужны более длительные временные периоды для выявления соответствующих долгосрочных тенденций относительной конвергенции для стран, вступивших в ЕС. Для группы стран, присоединившихся первыми, доступно 34 года наблюдений, но этот период сокращается до 26 лет для стран второго и до 12 лет для последнего расширения ЕС. Для четкого разграничения краткосрочных колебаний вокруг 
средних значений и долгосрочных тенденций конвергенции/дивергенции, особенно в случае их перекрещивания, возможно, необходим более длинный временной ряд.

Важно и то, что различия могут быть настолько незначительными, что их дальнейшего сокращения будет трудно достичь. При показателях, опустившихся ниже определенного порога, страны могут рассматриваться, по крайней мере, частично, как достигшие конвергенции. Это утверждение, которое касается как абсолютной, так и относительной конвергенции, требует более глубокого анализа и может быть в дальнейшем проверено с помощью анализа условной конвергенции.



Рисунок 10. Показатели рождаемости в странах ЕC-12, 1960-2007 гг. 

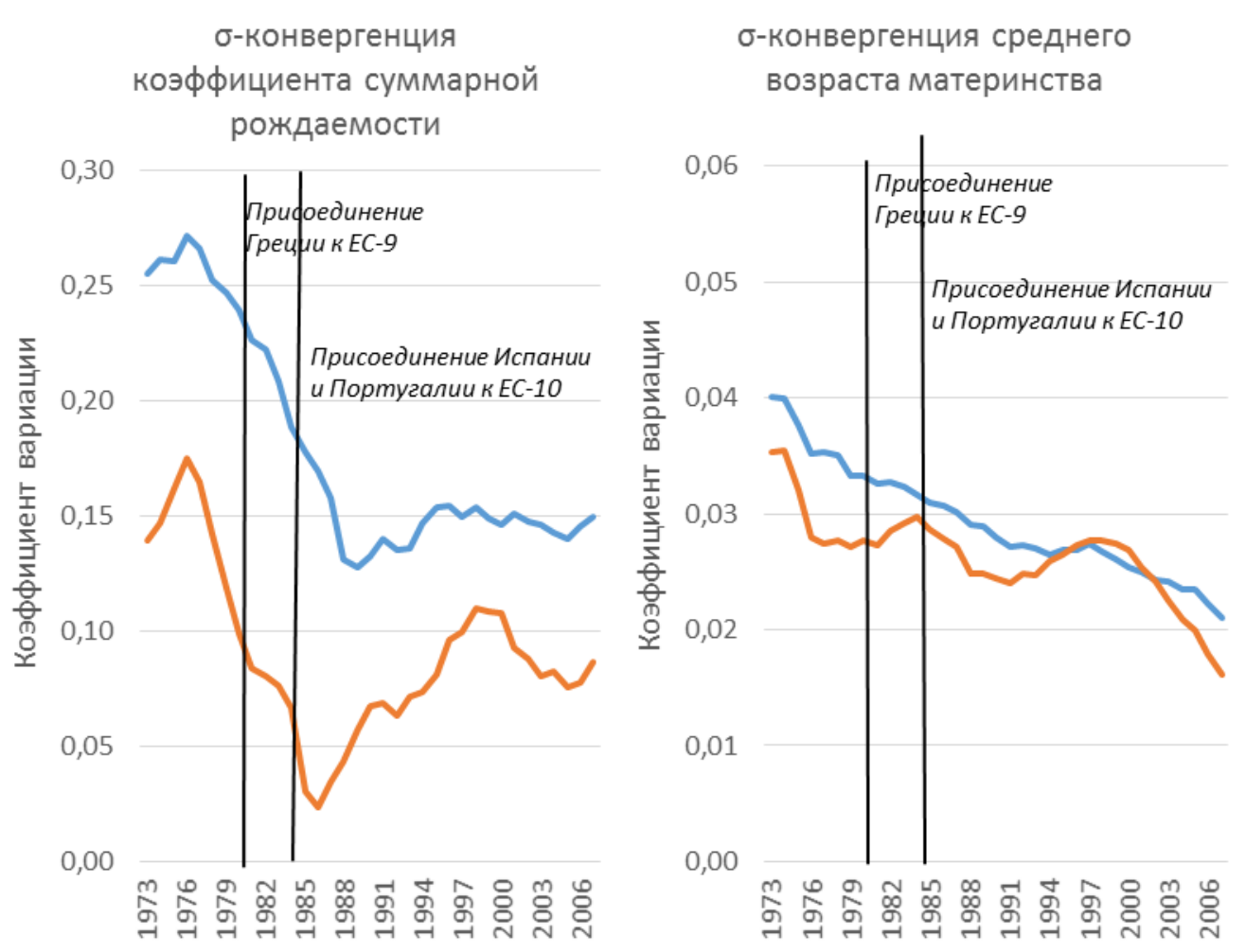

Рисунок 11. б-конвергенция рождаемости в странах ЕC-12, 1973-2007 гг.
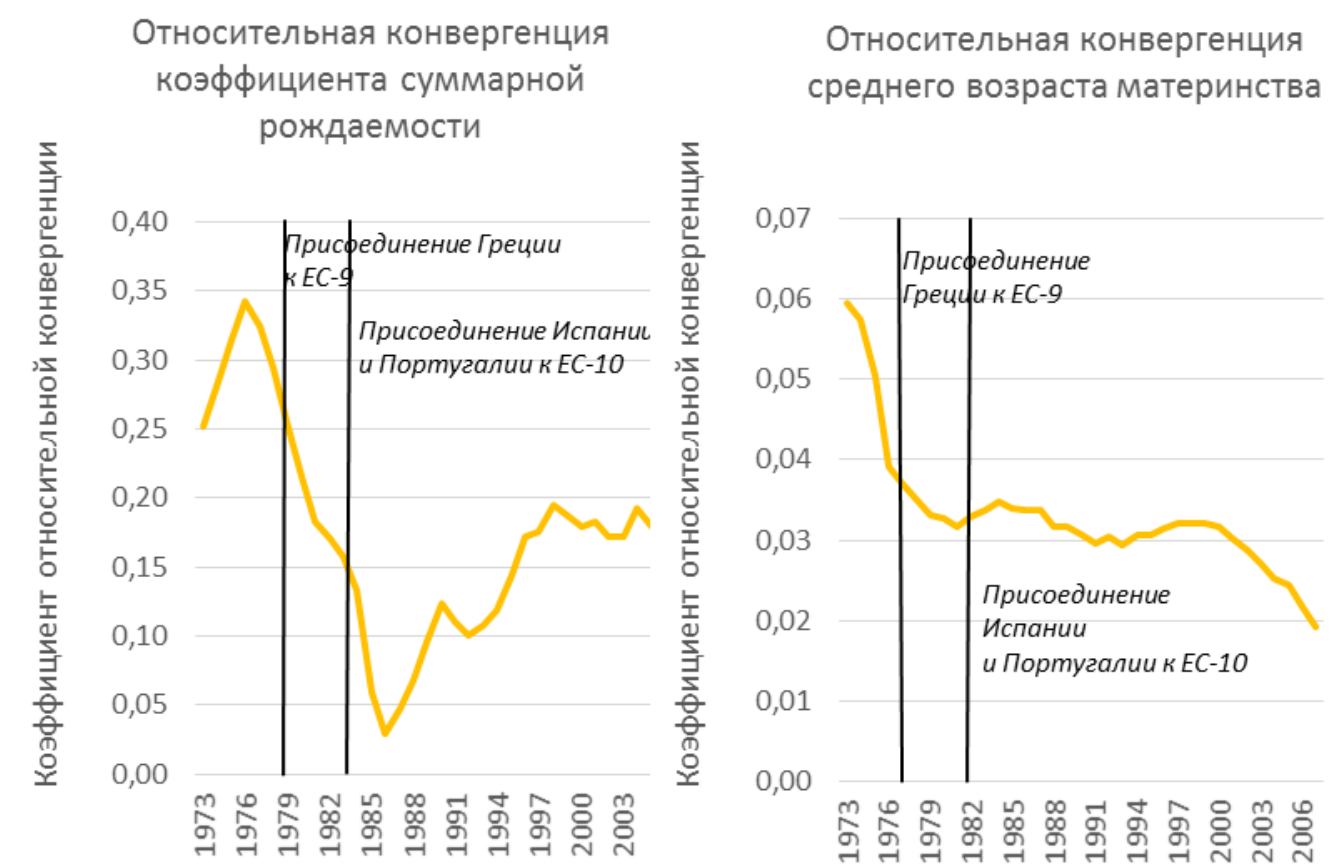

Рисунок 12. Относительная конвергенция показателей рождаемости Греции, Испании и Португалии с показателями стран ЕС- 9, 1973-2007 гг. 


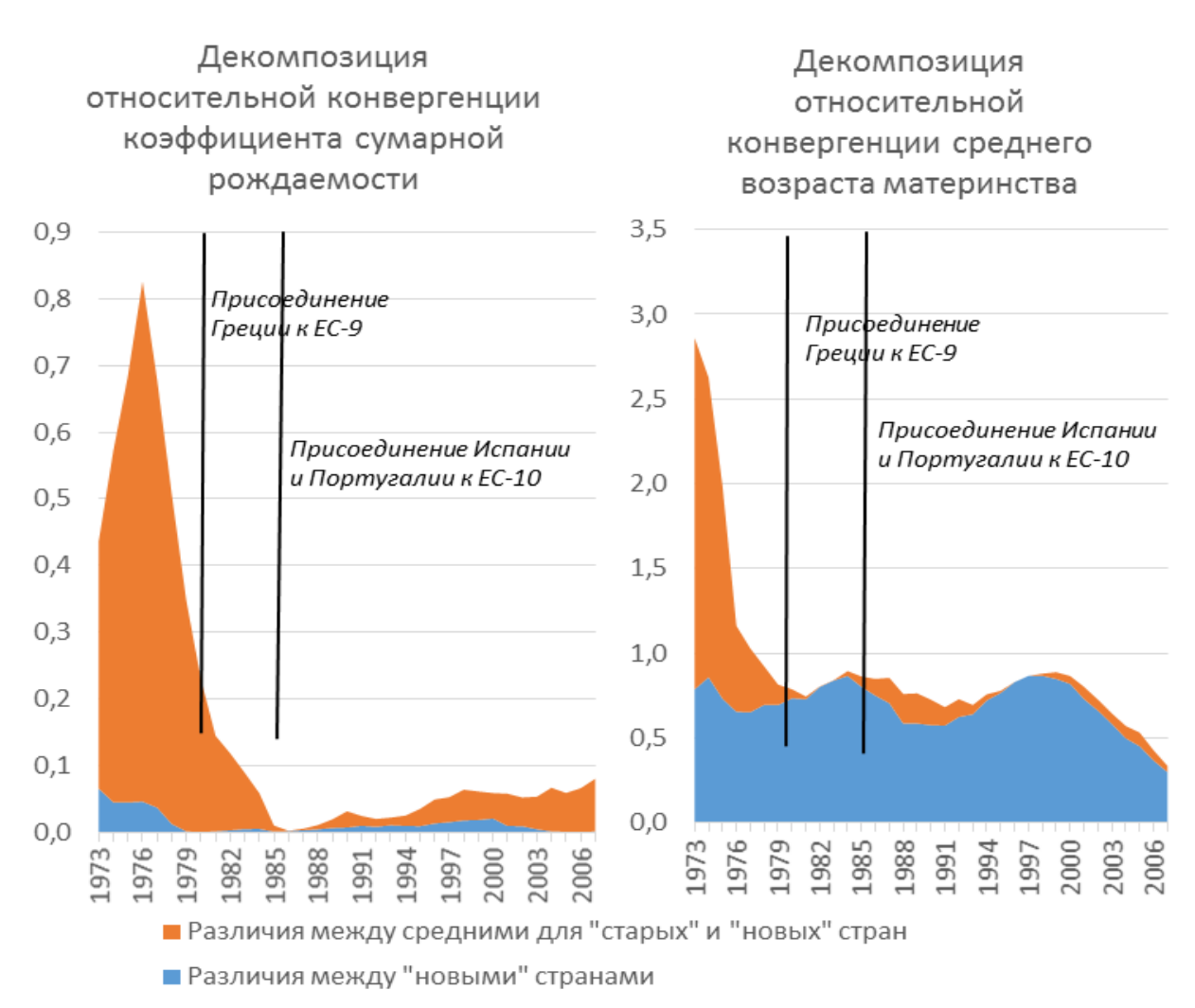

Рисунок 13. Декомпозиция относительной конвергенции показателей рождаемости Греции, Испании и Португалии с показателями стран ЕС-9, 1973-2007 гг.

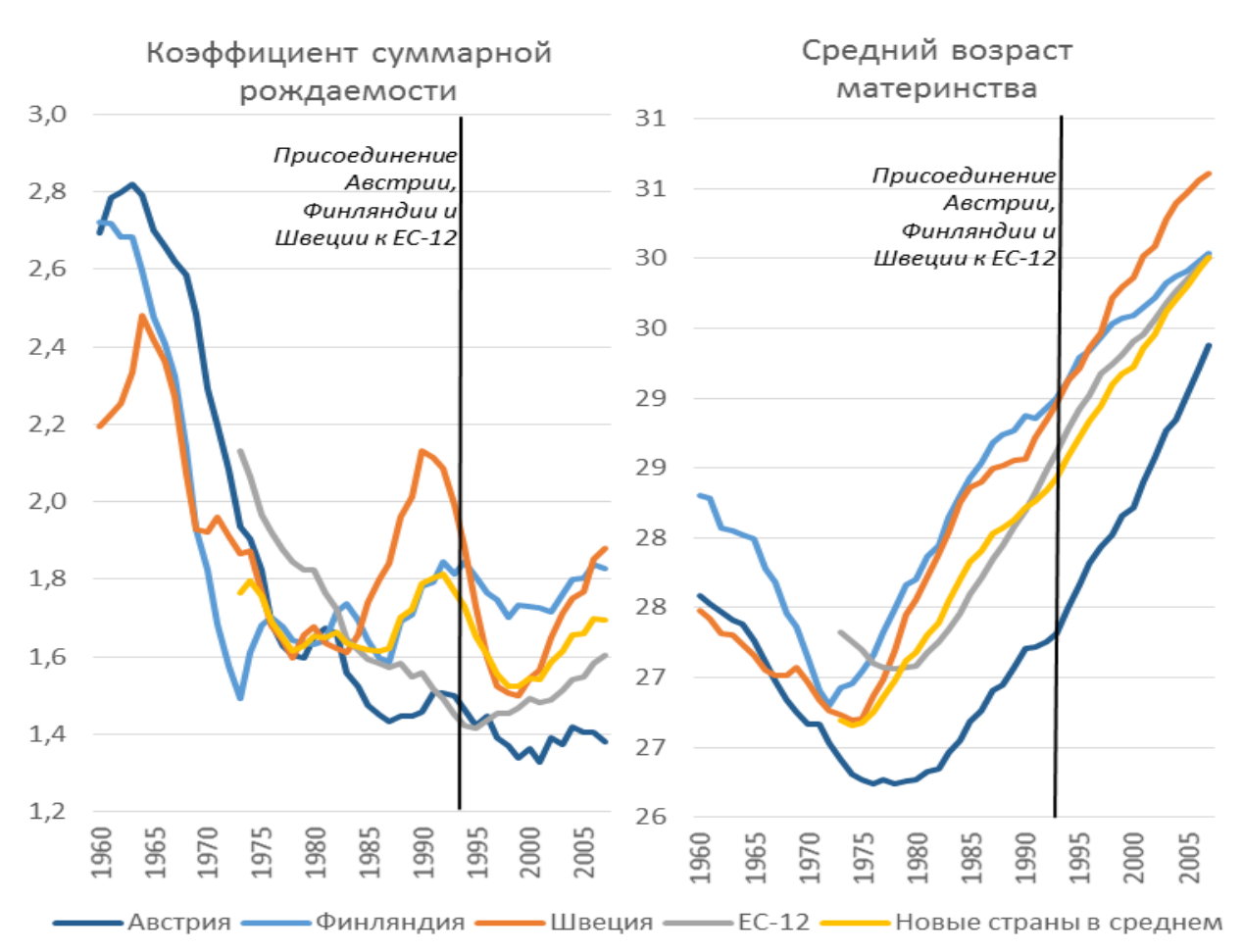

Рисунок 14. Показатели рождаемости в странах ЕС- 15, 1960-2007 гг. 


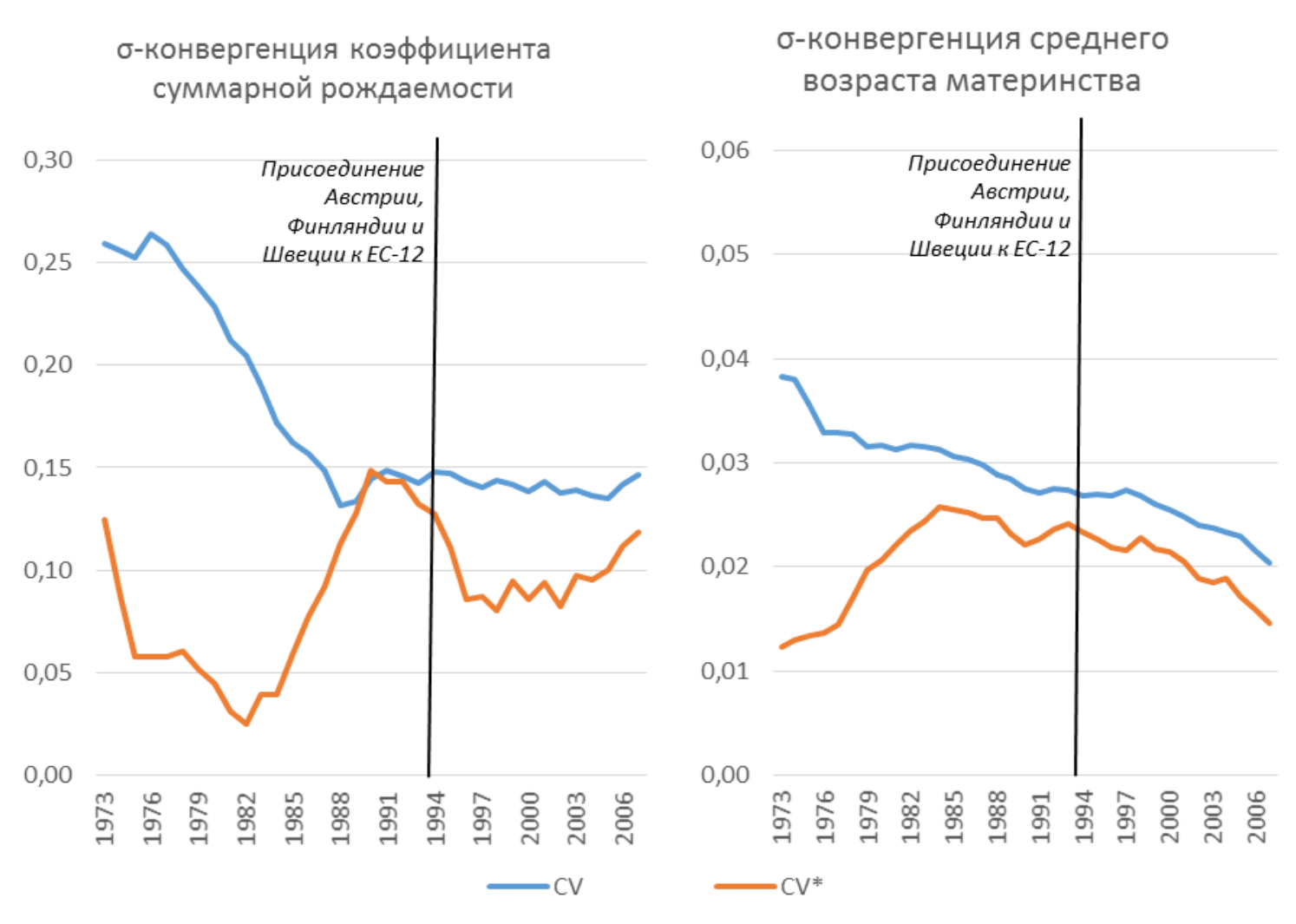

Рисунок 15. б-конвергенция показателей рождаемости в странах ЕС-15, 1973-2007 гг.
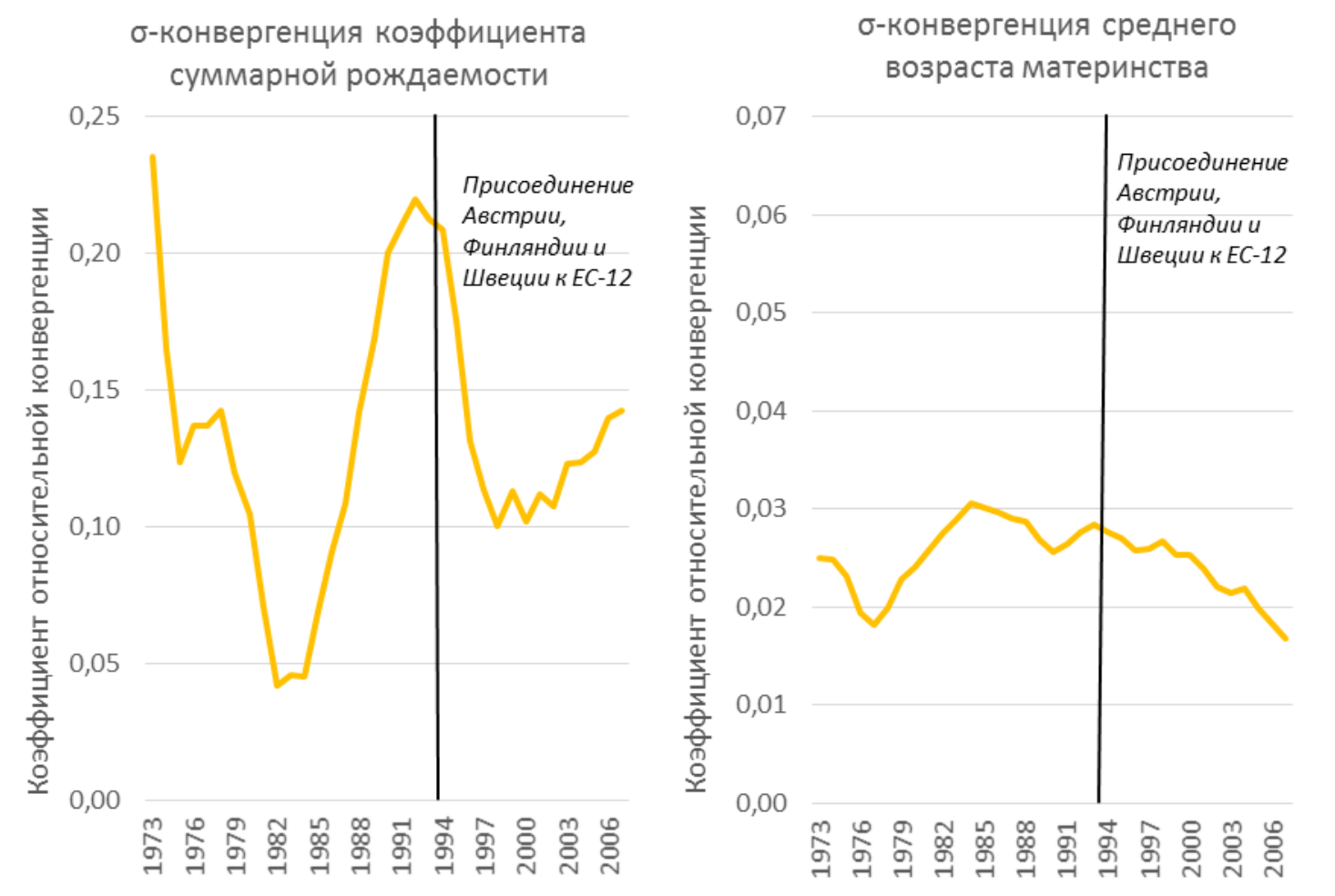

Рисунок 16. Относительная конвергенция показателей рождаемости Австрии, Финляндии и Швеции со странами ЕС- 12, 1973-2007 гг. 


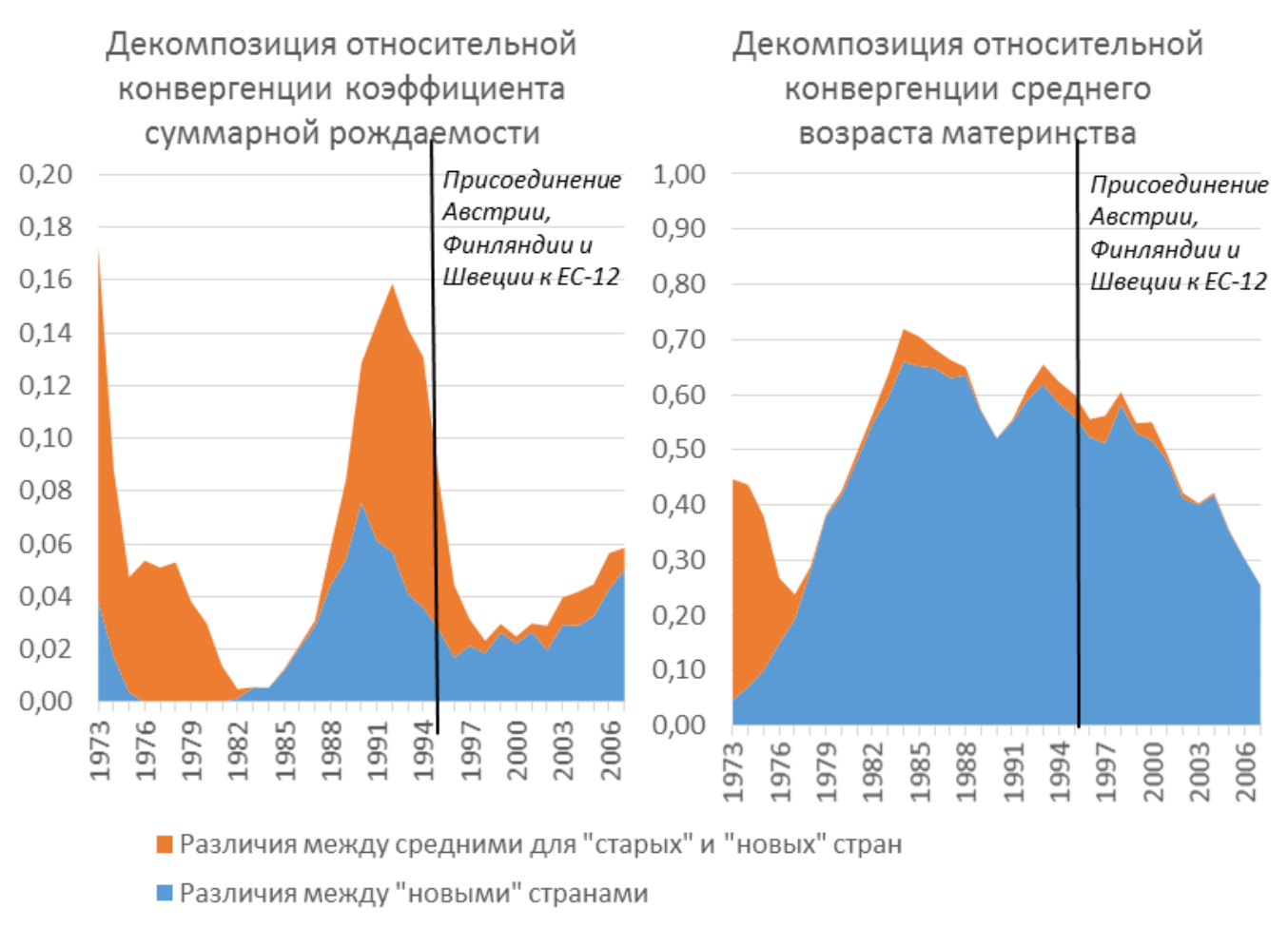

Рисунок 17. Декомпозиция относительной конвергенции показателей рождаемости Австрии, Финляндии и Швеции с показателями стран ЕС-12, 1973-2007 гг.

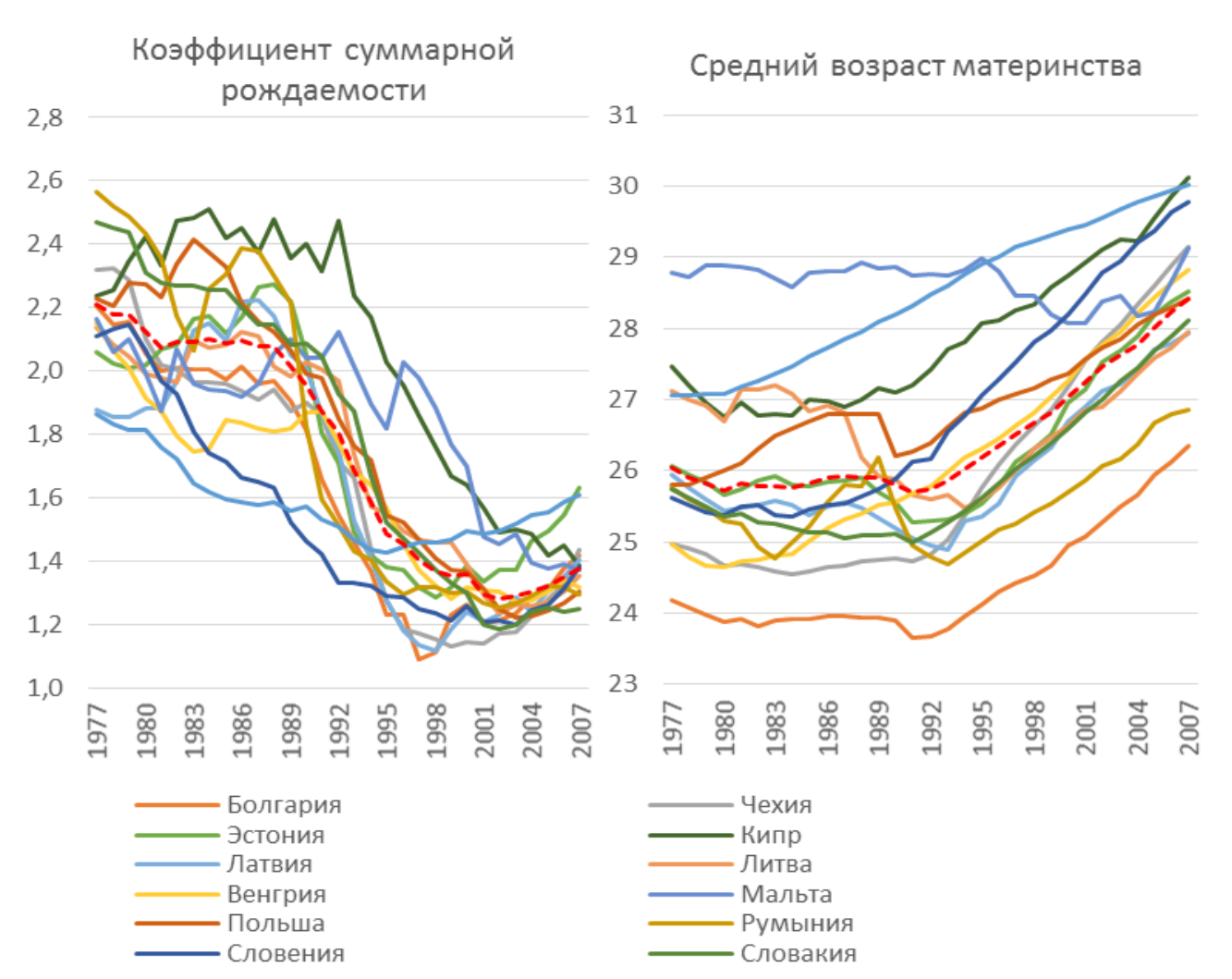

Рисунок 18. Показатели рождаемости в странах ЕС- 27, 1977-2007 гг. 


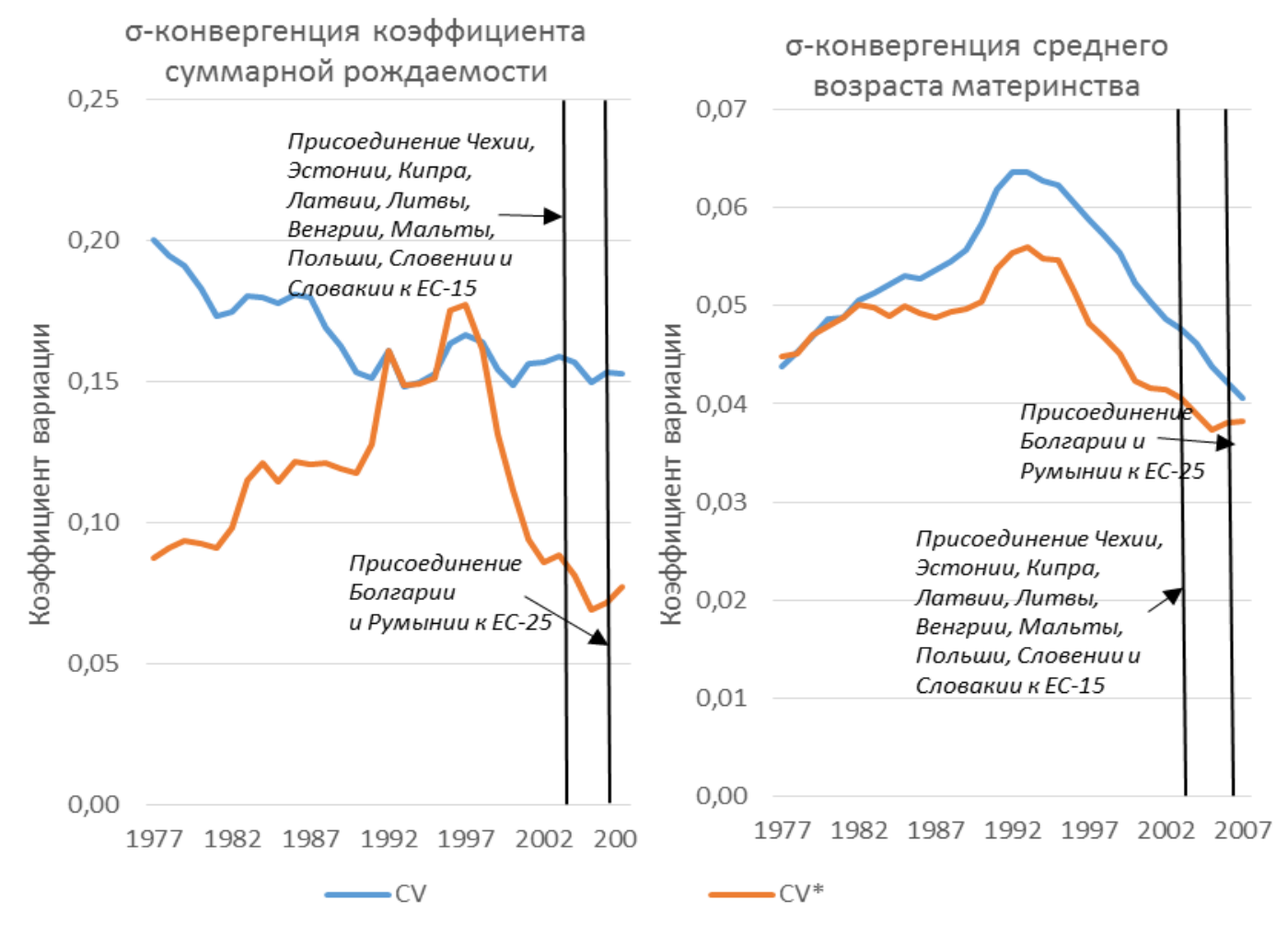

Рисунок 19. б-конвергенция показателей рождаемости в странах ЕС-27, 1977-2007 гг.


Рисунок 20. Относительная конвергенция показателей рождаемости Болгарии, Венгрии, Кипра, Латвии, Литвы, Мальты, Польши, Румынии, Словакии, Словении, Чехии и Эстонии с показателями стран ЕС- 15, 1977-2007 гг. 


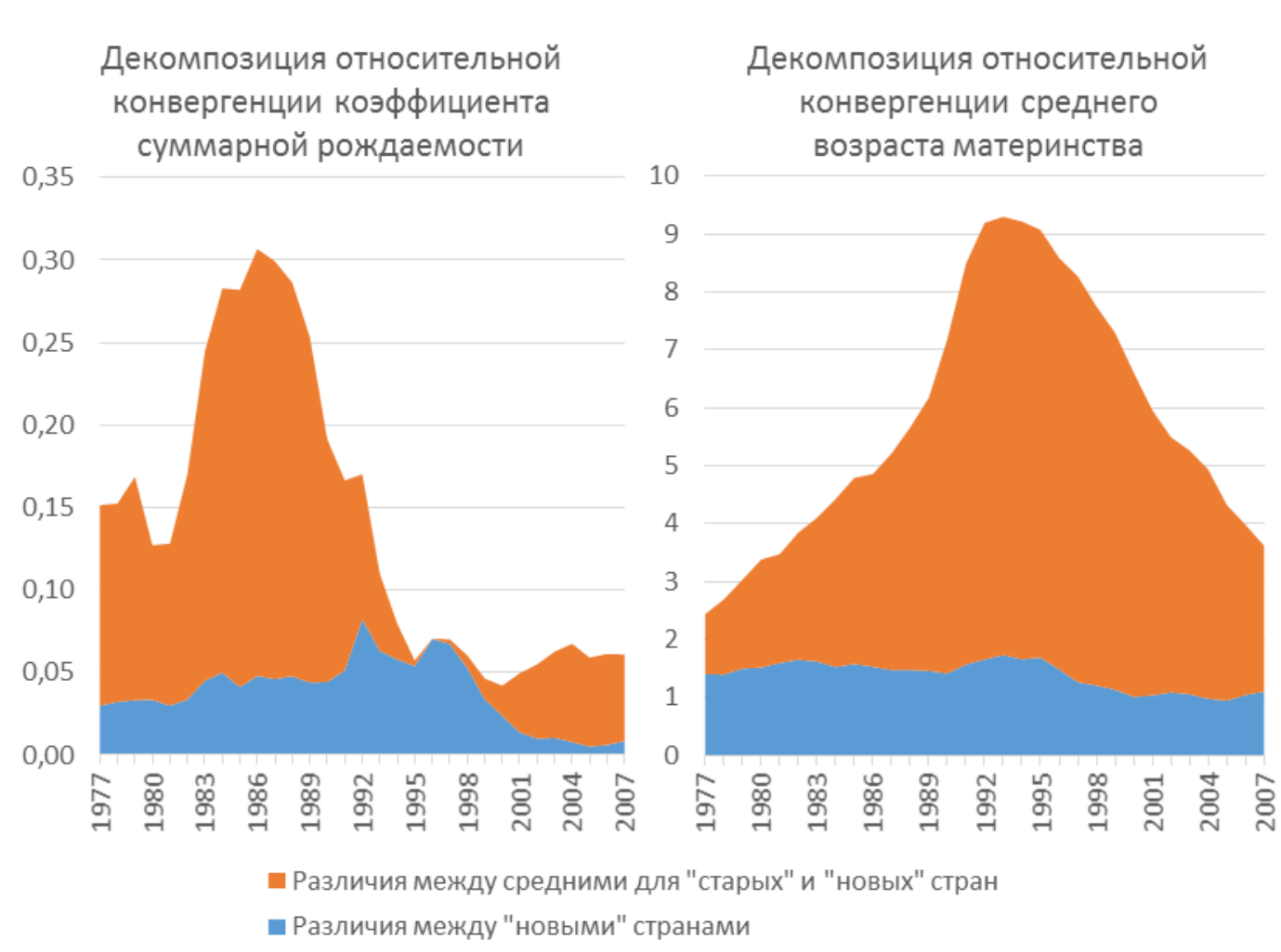

Рисунок 21. Декомпозиция относительной конвергенции показателей рождаемости Болгарии, Венгрии, Кипра, Латвии, Литвы, Мальты, Польши, Румынии, Словакии, Словении, Чехии и Эстонии с показателями стран ЕС-15, 1977-2007 гг.

\section{6 . ЗАКЛЮЧЕНИЕ}

В этой статье я обращаю внимание на необходимость учитывать при разработке прогнозных гипотез для группы стран дополнительных ограничений, накладываемых взаимной согласованностью ожидающих их изменений. В случае рождаемости она может быть выражена в виде конвергенции демографического поведения стран в будущем, что должно найти отражение в прогнозных гипотезах. Такое «международное» ограничение может иметь как теоретические, так и эмпирические основания.

С теоретической точки зрения это может быть связано с теорией демографического перехода; для эмпирических обоснований необходимо определить понятие конвергенции в операциональных терминах. Отталкиваясь от обширной литературы, посвященной проблеме экономической конвергенции, и используя три наиболее распространенные измерителя ( $\sigma$-, $\beta$ - и $\gamma$-конвергенцию), я прежде всего предположил наличие абсолютной конвергенции рождаемости. Для того чтобы учесть не только интенсивность, но и тайминг рождаемости, я использовал два показателя, пригодные для международных сравнений: коэффициент суммарной рождаемости и средний возраст матери при рождении ребенка. Анализ, охватывающий более чем 30-летний период для 27 европейских стран, показывает, что конвергенция рождаемости происходила и в той, и в другой форме, хотя временами наблюдались и периоды дивергенции. Тем не менее явных эмпирических доказательств, 
указывающих на будущую эволюцию, нет, поскольку разброс КСР в самое последнее время практически постоянен, а конвергенция СВМ, хотя и в самом деле имеет место, но после периода дивергенции, сходной по интенсивности и продолжительности с конвергенцией нынешнего периода. Это указывает на то, что абсолютная конвергенция может рассматриваться как ограничение, которое следует учитывать при разработке гипотез, но это должно получить также и теоретическое обоснование. Поэтому я проверяю дополнительную гипотезу, согласно которой членство в ЕС играет ту или иную роль в демографическом поведении на национальном уровне. Эта гипотеза предполагает допущение, что существуют общие ценности, разделяемые странами ЕС, к сближению с которыми могут двигаться вновь вступившие страны. Я полагаю, что измерители, используемые в литературе (во всяком случае, самые простые из них), недостаточны для целей исследования этого предположения, поэтому предлагаю и использую показатель относительной конвергенции. С помощью этого понятия я хочу подчеркнуть идею движения в направлении некоторого значения (определенного теоретически или зафиксированного эмпирически в какой-то группе стран), что отличается от конвергенции между странами. Если такая относительная конвергенция имела место в прошлом, это может служить дополнительным доводом в пользу предположения о конвергенции рождаемости среди стран ЕС. Чтобы проверить эту гипотезу, я анализирую расширения ЕС, происходившие в 1973, в 1981-1986 и в 1995 г., используя показатель, который я называю «коэффициентом относительной конвергенции». Общий итог несколько размыт, так как я не получил исчерпывающих доказательств сближения показателей рождаемости новых государств-членов со значениями, общими для ЕС. Тем не менее такие аргументы, как ограниченность временных рядов, используемых для анализа, а также определенные политические усилия и обмен лучшими практиками и опытом в области рождаемости в ЕС могли бы поддержать идею, что конвергенция рождаемости между членами ЕС может использоваться как правдоподобная гипотеза при прогнозировании на уровне ЕС.

В этой статье не было попытки изучения условной конвергенции или клубной конвергенции в ЕС. Ничего также не сказано о тайминге конвергенции или о значениях, к которым будет стремиться конвергенция. Изучению первого вопроса, однако, может помочь инструмент, предложенный в этой статье. Если предположить, что значение конвергенции определено на теоретическом уровне, наблюдаемые временные ряды коэффициентов относительной конвергенции могут быть использованы для оценки скорости конвергенции. Например, такой временной ряд мог бы быть должным образом экстраполирован, чтобы вычислить, когда прогнозное значение становится нулем.

Кроме того, возможность декомпозиции коэффициента относительной конвергенции позволила бы оценить, происходит ли эта конвергенция в различиях внутри распределения, в различиях между средним значением и значением, к которому ведет конвергенция, или только в одном из этих компонент. Ведь страны могут сохранять определенные различия между собой, в то время как их среднее значение сходится к теоретическому. Если сформулированы гипотезы в отношении КСР и СВМ, то с учетом нескольких дополнительных гипотез может быть разработана полная референтная модель рождаемости ко времени завершения конвергенции [Schmertmann 2003; 2005] и из этого 
может быть выведена вся эволюция рождаемости от базового года до конца прогноза [Lanzieri 2009].

\section{ЛИТЕРАТУРА}

Abreu M., H.L.F. de Groot, R.J.G.M. Florax (2005). A meta-analysis of beta-convergence: the legendary two-percent // Discussion Paper TI 2005-001/3. Amsterdam-Rotterdam: Tinbergen Institute.

Azzoni C., N. Menezes-Filho, T. Menezes (2003). Opening the convergence black box // Discussion Paper. № 2003/56. WIDER — World Institute for Development Economics Research, United NationsUniversity.

Barro R., X. Sala-i-Martin (1992). Convergence // Journal of Political Economy. 100: 223-51.

Boyle G.E., T.G. McCarthy (1997). A simple measure of beta-convergence // Oxford Bulletin of Economics and Statistics. 59(2): 257-264.

Boyle G.E., T.G. McCarthy (1999). Simple measures of convergence in per capita GDP: a note on some further international evidence // Applied Economics Letters. 6: 343-347.

Cole M.A., E. Neumayer (2003). The pitfalls of convergence analysis: is the income gap really widening? // Applied Economics Letters. 10:355-357.

Coleman D. (2004). Why we don't have to believe without doubting in the "Second Demographic Transition" - some agnostic comments // Vienna Yearbook for Population Research. 11-4.

Draxler J., O. Van Vliet (2010). European Social Model: No Convergence from the East // European Integration. 32(1): 115-135.

Dorius S.F. (2008). Global Demographic Convergence? A Reconsideration of Changing Intercountry Inequality in Fertility // Population and Development Review. 34(3): 519-537.

Franklin R.S. (2002). Fertility Convergence Across Italy’s Regions, 1952-1995 // Paper presented at the 41st Annual Meeting, Western Regional Science Association, Monterey, CA. February 2002.

Franklin R.S. (2003). Italian Fertility, 1864 to 1961: An Analysis of Regional Trends // Prepared for the 43rd European Congress of the Regional Science Association. Jyväskylä, Finland. 2730 August 2003.

Friedman M. (1992). Do Old Fallacies Ever Die? // Journal of Economic Literature. XXX: 21292132.

Haines M.R. (2002). Ethnic differences in demographic behaviour in the United States: has there been convergence? // Working Paper 9042, National Bureau of Economic Research. Cambridge (MA).

Herbertsson T.T., J.M. Orszag, P.R. Orszag (2000). Population Dynamics and Convergence in Fertility Rates // Working Papers in Economics \& Finance. Birkbeck, University of London.

Kaelble H. (1990). A Social History of Western Europe, 1880-1980. Barnes and Noble.

Kotzamanis B., M.-N. Duquenne (2006). Les disparités démographiques en Grèce, convergence on divergence?' // Balkan Demographic Paper. Vol. 7. Demobalk Network. 
Lanzieri G. (2009). EUROPOP2008: a set of population projections for the European Union // Paper for the XXVI IUSSP International Population Conference. Marrakech. October 2009.

Laurini M., E. Andrade, P.L. Valls Pereira (2005). Income convergence clubs for Brazilian municipalities: a non-parametric analysis // Applied Economics. 37: 2099-2118.

Maeso-Fernandez F. (2003). A time series approach to $\beta$-convergence // Applied Economics. 35: 1133-1146.

Phillips P.C.B., D. Sul (2007). Transition modelling and econometric convergence tests // Econometrica. 75(6): 1771-1855.

Preston S.H., P. Heuveline, M. Guillot (2001). Demography — Measuring and Modelling Population Processes. Blackwell Publishers.

Quah D. (1993). Galton's Fallacy and Tests of Convergence Hypothesis // Scandinavian Journal of Economics. 95(4): 427-443.

Sala-i-Martin X. (1994). Regional Cohesion: Evidence and Theories of Regional Growth and Convergence // Economics Working Paper 104. Department of Economics and Business, Universitat Pompeu Fabra.

Sala-i-Martin X. (1995). The Classical Approach to Convergence Analysis // Economics Working Paper 117. Department of Economics and Business, Universitat Pompeu Fabra.

Schmertmann C. (2003). A system of model fertility schedules with graphically intuitive parameters // Demographic Research. 9: 81-110.

Schmertmann C. (2005). Quadratic spline fits by nonlinear least squares // Demographic Research. 12: 105-106.

Tomljanovich M., T.J. Vogelsang (2002). Are U.S. regions converging? Using new econometric methods to examine old issues // Empirical Economics. 27: 49-62.

Tomka B. (2002). Demographic Diversity and Convergence in Europe, 1918-1990: The Hungarian Case // Demographic Research. 6: 19-48.

Vallin J., G. Caselli (2006). The Hypothetical Cohort as a Tool for Demographic Analysis // Caselli G., J. Vallin, and G. Wunsch, eds. Demography: Analysis and Synthesis. Vol. I, chap. 14: 163-195. Academic Press.

Van de Kaa D.J. (2004). Is the Second Demographic Transition a useful research concept? Questions and answers // Vienna Yearbook for Population Research. 4-10.

Watkins S.C. (1990). From Provinces into Nations: Demographic Integration in Western Europe, 1870- 1960. Princeton University Press.

Wilson C. (2001). On the Scale of Global Demographic Convergence 1950-2000 // Population and Development Review. 27: 155-171.

Young A.T., M.J. Higgins, D. Levy (2008). Sigma Convergence versus Beta Convergence: Evidence from U.S. County-Level Data // Journal of Money, Credit and Banking. 40: 10831093. 


\title{
IS FERTILITY CONVERGING ACROSS THE MEMBER STATES OF THE EUROPEAN UNION? *
}

\author{
GIAMPAOLO LANZIERI
}

GiAMPAOlo LANZIERI, EUROPEAN COMMISSION — EUROSTAT, POPULATION UNIT. LUXEMBOURG.

E-MAIL: giampaolo.lanzieri@ec.europa.eu.

THE VIEWS EXPRESSED IN THIS PAPER ARE EXCLUSIVELY THOSE OF THE AUTHOR AND DO NOT NECESSARILY REPRESENT THE VIEWS OF THE EUROPEAN COMMISSION.

\begin{abstract}
The article shows the importance of taking into account additional constraints of consistency when preparing the projections assumptions for a set of countries. In the case of fertility, described in this article, this may be expressed in the form of convergence between countries with which future national demographic behavior could be required - in the assumptions setting — to comply. This "international" constraint may have both theoretical and empirical grounds.

The paper presents an analysis of the various concepts of convergence proposed in the literature together with the related indicators. The author's method of analysis and a new simple indicator of convergence are defined, and are used to assess the presence of convergence in the whole set of 27 countries currently belonging to the European Union. The author analyzes the possible impact of accession to the European Union on convergence in fertility for some of the EU enlargements that have occurred in the past. Finally, the implications for making assumptions in the projections exercises are discussed.
\end{abstract}

Key words: fertility convergence, European Union, EU enlargement, projections assumptions in fertility.

* Original article Lanzieri Giampaolo (2010). Is Fertility conVerging aCross the Member States of the EUROPEAN UNION? // WORK SESSION ON DEMOGRAPHIC PROJECTIONS. LISBON, 28-30 APRIL 2010. LUXEMBOURG:

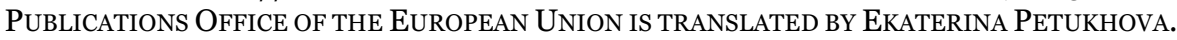

\section{REFERENCES}

Abreu M., H.L.F. de Groot, R.J.G.M. Florax (2005). A meta-analysis of beta-convergence: the legendary two-percent // Discussion Paper TI 2005-001/3. Amsterdam-Rotterdam: Tinbergen Institute.

Azzoni C., N. Menezes-Filho, T. Menezes (2003). Opening the convergence black box // Discussion Paper. № 2003/56. WIDER — World Institute for Development Economics Research, United NationsUniversity.

Barro R., X. Sala-i-Martin (1992). Convergence // Journal of Political Economy. 100: 223-51.

Boyle G.E., T.G. McCarthy (1997). A simple measure of beta-convergence // Oxford Bulletin of Economics and Statistics. 59(2): 257-264.

Boyle G.E., T.G. McCarthy (1999). Simple measures of convergence in per capita GDP: a note on some further international evidence // Applied Economics Letters. 6: 343-347.

Cole M.A., E. Neumayer (2003). The pitfalls of convergence analysis: is the income gap really widening? // Applied Economics Letters. 10:355-357.

Coleman D. (2004). Why we don't have to believe without doubting in the "Second Demographic Transition" - some agnostic comments // Vienna Yearbook for Population Research. 11-4. 
Draxler J., O. Van Vliet (2010). European Social Model: No Convergence from the East // European Integration. 32(1): 115-135.

Dorius S.F. (2008). Global Demographic Convergence? A Reconsideration of Changing Intercountry Inequality in Fertility // Population and Development Review. 34(3): 519-537.

Franklin R.S. (2002). Fertility Convergence Across Italy's Regions, 1952-1995 // Paper presented at the 41st Annual Meeting, Western Regional Science Association, Monterey, CA. February 2002.

Franklin R.S. (2003). Italian Fertility, 1864 to 1961: An Analysis of Regional Trends // Prepared for the 43rd European Congress of the Regional Science Association. Jyväskylä, Finland. 2730 August 2003.

Friedman M. (1992). Do Old Fallacies Ever Die? // Journal of Economic Literature. XXX: 21292132.

Haines M.R. (2002). Ethnic differences in demographic behaviour in the United States: has there been convergence? // Working Paper 9042, National Bureau of Economic Research. Cambridge (MA).

Herbertsson T.T., J.M. Orszag, P.R. Orszag (2000). Population Dynamics and Convergence in Fertility Rates // Working Papers in Economics \& Finance. Birkbeck, University of London.

Kaelble H. (1990). A Social History of Western Europe, 1880-1980. Barnes and Noble.

Kotzamanis B., M.-N. Duquenne (2006). Les disparités démographiques en Grèce, convergence on divergence?' // Balkan Demographic Paper. Vol. 7. Demobalk Network.

Lanzieri G. (2009). EUROPOP2008: a set of population projections for the European Union // Paper for the XXVI IUSSP International Population Conference. Marrakech. October 2009.

Laurini M., E. Andrade, P.L. Valls Pereira (2005). Income convergence clubs for Brazilian municipalities: a non-parametric analysis // Applied Economics. 37: 2099-2118.

Maeso-Fernandez F. (2003). A time series approach to $\beta$-convergence // Applied Economics. 35: 1133-1146.

Phillips P.C.B., D. Sul (2007). Transition modelling and econometric convergence tests // Econometrica. 75(6): 1771-1855.

Preston S.H., P. Heuveline, M. Guillot (2001). Demography — Measuring and Modelling Population Processes. Blackwell Publishers.

Quah D. (1993). Galton's Fallacy and Tests of Convergence Hypothesis // Scandinavian Journal of Economics. 95(4): 427-443.

Sala-i-Martin X. (1994). Regional Cohesion: Evidence and Theories of Regional Growth and Convergence // Economics Working Paper 104. Department of Economics and Business, Universitat Pompeu Fabra.

Sala-i-Martin X. (1995). The Classical Approach to Convergence Analysis // Economics Working Paper 117. Department of Economics and Business, Universitat Pompeu Fabra.

Schmertmann C. (2003). A system of model fertility schedules with graphically intuitive parameters // Demographic Research. 9: 81-110.

Schmertmann C. (2005). Quadratic spline fits by nonlinear least squares // Demographic Research. 12: 105-106. 
Tomljanovich M., T.J. Vogelsang (2002). Are U.S. regions converging? Using new econometric methods to examine old issues // Empirical Economics. 27: 49-62.

Tomka B. (2002). Demographic Diversity and Convergence in Europe, 1918-1990: The Hungarian Case // Demographic Research. 6: 19-48.

Vallin J., G. Caselli (2006). The Hypothetical Cohort as a Tool for Demographic Analysis // Caselli G., J. Vallin, and G. Wunsch, eds. Demography: Analysis and Synthesis. Vol. I, chap. 14: 163-195. Academic Press.

Van de Kaa D.J. (2004). Is the Second Demographic Transition a useful research concept? Questions and answers // Vienna Yearbook for Population Research. 4-10.

Watkins S.C. (1990). From Provinces into Nations: Demographic Integration in Western Europe, 1870- 1960. Princeton University Press.

Wilson C. (2001). On the Scale of Global Demographic Convergence 1950-2000 // Population and Development Review. 27: 155-171.

Young A.T., M.J. Higgins, D. Levy (2008). Sigma Convergence versus Beta Convergence:

Evidence from U.S. County-Level Data // Journal of Money, Credit and Banking. 40: 10831093. 